\title{
Yap/Taz regulate alveolar regeneration and resolution of lung inflammation
}

\author{
Ryan LaCanna, ${ }^{1}$ Daniela Liccardo,, ${ }^{1}$ Peggy Zhang, ${ }^{1}$ Lauren Tragesser, ${ }^{1}$ Yan Wang, ${ }^{2}$ Tongtong Cao, ${ }^{1}$ Harold A. Chapman, ${ }^{3}$ \\ Edward E. Morrisey, ${ }^{4}$ Hao Shen, ${ }^{2}$ Walter J. Koch, ${ }^{1}$ Beata Kosmider, ${ }^{5}$ Marla R. Wolfson, ${ }^{5}$ and Ying Tian ${ }^{1}$ \\ 'Department of Pharmacology, Center for Translational Medicine, Temple University Lewis Katz School of Medicine, Philadelphia, Pennsylvania, USA. Department of Microbiology, Perelman School \\ of Medicine, University of Pennsylvania, Philadelphia, Pennsylvania, USA. ${ }^{3}$ Department of Medicine, Cardiovascular Research Institute, UCSF, San Francisco, California, USA. ${ }^{4}$ Department of Medicine, \\ Department of Cell and Developmental Biology, Perelman School of Medicine, University of Pennsylvania, Philadelphia, Pennsylvania, USA. ${ }^{5}$ Department of Physiology, Department of Thoracic Medicine and \\ Surgery, Center for Inflammation, Translational and Clinical Lung Research, Temple University Lewis Katz School of Medicine, Philadelphia, Pennsylvania, USA.
}

\begin{abstract}
Alveolar epithelium plays a pivotal role in protecting the lungs from inhaled infectious agents. Therefore, the regenerative capacity of the alveolar epithelium is critical for recovery from these insults in order to rebuild the epithelial barrier and restore pulmonary functions. Here, we show that sublethal infection of mice with Streptococcus pneumoniae, the most common pathogen of community-acquired pneumonia, led to exclusive damage in lung alveoli, followed by alveolar epithelial regeneration and resolution of lung inflammation. We show that surfactant protein C-expressing (SPC-expressing) alveolar epithelial type II cells (AECIIs) underwent proliferation and differentiation after infection, which contributed to the newly formed alveolar epithelium. This increase in AECII activities was correlated with increased nuclear expression of Yap and Taz, the mediators of the Hippo pathway. Mice that lacked Yap/Taz in AECIls exhibited prolonged inflammatory responses in the lung and were delayed in alveolar epithelial regeneration during bacterial pneumonia. This impaired alveolar epithelial regeneration was paralleled by a failure to upregulate ІкBa, the molecule that terminates NF-кB-mediated inflammatory responses. These results demonstrate that signals governing resolution of lung inflammation were altered in Yap/Taz mutant mice, which prevented the development of a proper regenerative niche, delaying repair and regeneration of alveolar epithelium during bacterial pneumonia.
\end{abstract}

\section{Introduction}

Bacterial pneumonia is a leading cause of mortality and morbidity worldwide. Despite effective antibiotics and vaccines, there is still substantial pulmonary morbidity in susceptible individuals (1). Infection by Streptococcus pneumoniae (the pneumococcus), the most common pathogen of community-acquired pneumonia, is characterized by acute elevation of bacterial number in the lung, diffuse alveolar epithelial damage, and a robust alveolar influx of leukocytes (2). While the virulence of the pneumococcus and host factors that contribute to bacterial pneumonia are becoming clear, up to now, little has been known about the types of damaged lung cells induced by bacterial pneumonia and the extent of lung regeneration following injury.

Models of lung damage in mice have demonstrated that the regenerative processes in the lung involve local stem/progenitor cell populations (3). Alveolar epithelial type II cells (AECIIs), which express surfactant protein C (SPC), have been considered to be stem/progenitor cells for the alveolar epithelium (3). Lineage tracing of $\mathrm{SPC}^{+} \mathrm{AECIIs} \mathrm{demonstrates} \mathrm{their} \mathrm{capacities} \mathrm{of} \mathrm{self-}$

Conflict of interest: The authors have declared that no conflict of interest exists. Copyright: (๑) 2019, American Society for Clinical Investigation.

Submitted: September 18, 2018; Accepted: March 5, 2019; Published: April 15, 2019. Reference information: / Clin Invest. 2019;129(5):2107-2122.

https://doi.org/10.1172/JCl125014. renewal and differentiation into AECIs during both the homeostatic state and regeneration after injury (4). Besides their stem/ progenitor capacities, AECIIs have immune-modulatory functions. AECIIs release cytokines and chemokines that recruit leukocytes to the sites of injury/infection and activate these cells to mount an immune response $(5,6)$. Dysregulated immune responses have been implicated in a variety of inflammatory and fibrotic conditions affecting humans. In some types of pulmonary fibrosis, patients have abnormal alveolar structure with a loss of AECIs and accumulation of immune cells (7-9). The mechanisms for the failure of AECI regeneration are not clear, however. One explanation for the loss of AECIs is the loss of local stem/progenitor cells, the sources for generating new AECIs, after injury. However, this stem/progenitor cell loss cannot be the sole reason for the impaired regeneration, as lung injuries that do not cause loss of stem/progenitor cells can still lead to failed AECI regeneration in animal models and patients with pulmonary fibrosis (10). Those findings imply that non-stem/progenitor cell loss-specific mechanisms are responsible for the impaired lung regeneration that occurs in fibrotic lungs.

The molecular mechanisms underlying lung regeneration have been studied in adult mice following pneumonectomy (11). Regeneration of lung alveoli depends on an increased inflammatory state in the lung, a process that requires recruitment of monocytes and macrophages into lung alveoli. It is unclear how inflammatory 
responses that normally lead to lung regeneration in acute injury are absent in fibrotic lungs with persistent inflammatory responses.

Here, we show that mice infected with $S$. pneumoniae strain $\mathrm{T} 4$ (SpT4) have injuries exclusively in lung alveoli, with loss of AECIs and AECIIs and increased infiltration of immune cells. This is followed by rapid resolution of lung inflammation and alveolar epithelial regeneration via proliferation of preexisting $\mathrm{SPC}^{+} \mathrm{AECIIs}$ and their differentiation into AECIs. We show that nuclear expression of Yap and Taz, the mediators of the Hippo signaling pathway, were markedly increased in AECIIs following SpT4 infection. We investigate the role of Yap and Taz in AECIIs during alveolar epithelial regeneration and resolution of lung inflammation in response to bacterial pneumonia-induced lung injury.

\section{Results}

Alveolar epithelial injury and recovery following S. pneumoniaeinduced bacterial pneumonia in mice. We used SpT4 to infect mice by intranasal inhalation under anesthetization with a dose of approximately $5 \times 10^{6} \mathrm{CFU}$. SpT 4 infection causes direct infection of the lower respiratory tract and acute bacterial pneumonia in mice, as indicated in previous reports $(12,13)$. Lungs were harvested at 2, 4, 7, and 14 days post infection (dpi) and analyzed by histology and flow cytometry. The levels of pneumococcal bacteria and cell apoptosis were highest in the alveolar spaces within the first 2 days after infection, as indicated by immunostaining with antibody specific for SpT4 capsule and TUNEL staining, individually (Figure 1, A-C). In contrast, their levels were reduced after $2 \mathrm{dpi}$, as seen by the gradual decrease in $\mathrm{SpT}^{+}$and $\mathrm{TUNEL}^{+}$numbers within the alveolar region by $14 \mathrm{dpi}$. To determine pneumococci clearance in the lung, bacterial loads were measured by plating lung homogenate. A transient increase of bacterial load was detected in mouse lung, with the highest level at $2 \mathrm{dpi}$ and $4 \mathrm{dpi}$, followed by a significant drop at $7 \mathrm{dpi}$ (Figure 1B). Substantial destruction of alveolar epithelial cells was observed, as evidenced by significant loss of cell type-specific markers for AECIs (T1a) and AECIIs (SPC) at 2, 4, and $7 \mathrm{dpi}$ (Figure 1A). By $14 \mathrm{dpi}$, the number of those cell populations returned to the basal level (Figure 1, D and E). The damage and recovery of lung epithelium during bacterial pneumonia were specific to the alveolar region, as there were no significant changes in the expression of airway epithelial cells, including club cells (CC10), ciliated cells (bTubulin IV), and basal cells (p63) (Supplemental Figure 1A; supplemental material available online with this article; https://doi.org/10.1172/JCI125014DS1).

Alveolar epithelial regeneration and inflammatory resolution during bacterial pneumonia. We first examined AECII proliferation after SpT4-induced lung injury. To determine DNA synthesis in AECIIs, mice received a single i.p. injection of EdU and were sacrificed after a 3-hour labeling period. The frequency of EdU incorporation was determined on sectioned lungs by colabeling with antibody against AECII (SPC) (Figure 2A). A transient increase of DNA synthesis was observed in AECIIs, with a peak labeling index of $7 \% \pm 0.3 \%$ occurring at $4 \mathrm{dpi}$ (Figure $2 \mathrm{~B}$ ).

We next performed genetic lineage tracing of adult $\mathrm{SPC}^{+}$ AECIIs using the SPC-CreERT2, Rosa26-mTmG mouse line. Mice were administrated with 3 i.p. injections of tamoxifen at $0.2 \mathrm{mg} / \mathrm{g}$ per dose to induce GFP expression in $\mathrm{SPC}^{+} \mathrm{AECIIs.} \mathrm{Mice} \mathrm{were} \mathrm{then}$ infected with SpT4 at 14 days after the last tamoxifen treatment.
Lungs were analyzed by immunohistochemistry and flow cytometry. Differentiation of AECIIs to AECIs was measured by coimmunostaining for lineage-labeled AECII marker (GFP) and AECI marker (T1a) (Figure 2C). We examined AECII-to-AECI differentiation by quantifying the percentage of $\mathrm{GFP}^{+}$alveolar surface area covered by AECII-derived AECIs $\left(\mathrm{GFP}^{+} \mathrm{T}^{+} \mathrm{a}^{+}\right)$on sectioned lungs. There were no significant changes between noninfected and SpT4-infected lungs within 6 dpi. However, we observed significant increases in the levels of AECII-to-AECI differentiation $\left(\mathrm{GFP}^{+} \mathrm{T} 1 \mathrm{a}^{+}\right)$at $7 \mathrm{dpi}$ and onwards (Figure 2D and Supplemental Figure 1B). Consistent with this finding, FACS analysis showed the significant increase in the percentage of AECIs derived from preexisting AECIIs $\left(\mathrm{GFP}^{+} \mathrm{Tla}^{+}\right)$at 7 dpi compared with that in noninfected lungs. At $14 \mathrm{dpi}$, the level of $\mathrm{GFP}^{+} \mathrm{T}^{+} \mathrm{a}^{+}$was elevated by 4.7 \pm 0.4 -fold compared with that at $7 \mathrm{dpi}$ (Figure 2E).

It has been postulated that inflammatory cells are important drivers of tissue repair and regeneration $(14,15)$. However, correlations between persistent lung inflammation and decreased lung regeneration have been observed in both various animal injury models and human patients $(8,10)$. To assess the lung inflammatory responses and their association with alveolar epithelial regeneration, we characterized the immune phenotypes in mouse lungs during bacterial pneumonia. Consistent with previous reports $(12,13)$, high levels of inflammatory responses were observed in the lung, as evidenced by the significant increases in the number of immune cells $\left(\mathrm{CD} 45^{+}\right)$within $7 \mathrm{dpi}$, including lymphocytes $\left(\mathrm{CD}^{+}\right)$, macrophages $\left(\mathrm{CD} 11 \mathrm{c}^{+} \mathrm{CD} 64^{+}\right)$, and neutrophils $\left(\mathrm{Ly}_{6 \mathrm{G}}{ }^{+}\right)$ (Supplemental Figure 2, A-E). Yet the accumulation of inflammatory cells in the lung waned over time, as seen by the decrease in the number of those cell populations by $14 \mathrm{dpi}$. Mouse lungs exhibited profibrotic lesions at $7 \mathrm{dpi}$, but recovered from SpT4 infection without the acquisition of lung fibrosis at $14 \mathrm{dpi}$, as evidenced by Masson's trichrome staining and hydroxyproline assay on lung tissue lysates (Supplemental Figure 2, F and G). The transient accumulation of lung inflammatory cells was accompanied by the loss of AECIs and the increase of profibrotic lesions, while the resolution of inflammatory cells coincided with the time frame of AECI recovery, AECII-to-AECI differentiation, and regression of profibrotic lesions in the lung (Supplemental Figure 2, $\mathrm{H}-\mathrm{J}$ ), implying that resolution of lung inflammation was closely associated with alveolar epithelial regeneration.

Yap and Taz expression in AECIIs during bacterial pneumonia. To understand the mechanisms underlying alveolar epithelial regeneration following SpT4-induced lung injury, we performed microarray analysis on lineage-labeled AECIIs $\left(\mathrm{GFP}^{+}\right)$isolated from SPC-CreERT2, Rosa26-mTmG mouse lungs before SpT4 infection and at 8 dpi with SpT4. After normalization of data sets, we used ToppGene to identify pathways that were induced (FDR, $<5 \%$ and $>1.5$-fold expression) in $\mathrm{GFP}^{+}$AECIIs of SpT4-infected mice. Pathways associated with Hippo signaling, $\mathrm{T}$ cell receptor signaling, JAK/STAT, and p38 signaling were all induced in SpT4-infected mice (Supplemental Table 1). Quantitative reverse transcriptase PCR (qRT-PCR) analysis confirmed increased expression of Yap and Taz, the 2 paralogous transcriptional coactivators of the Hippo pathway $(16,17)$, as well as their target genes including Ctgf, Cyr61, and Birc5 in GFP ${ }^{+}$AECIIs of SpT4-infected mice at 7 dpi compared with those of mice without infection $(0$ 
A
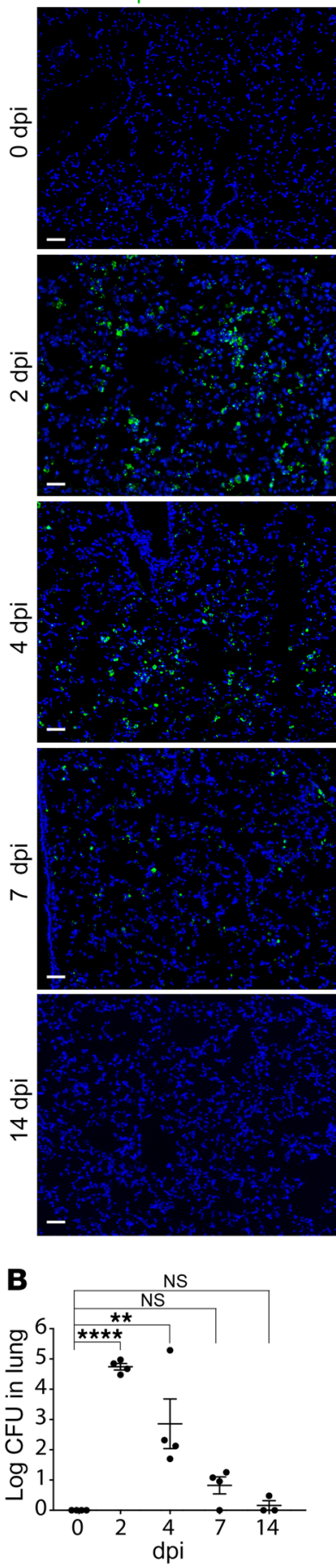

TUNEL/DAP
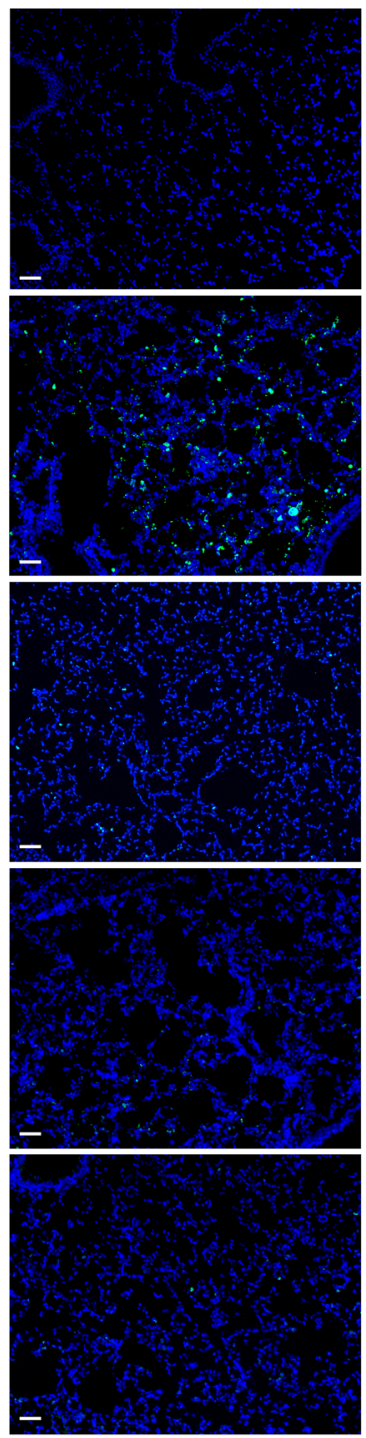

C

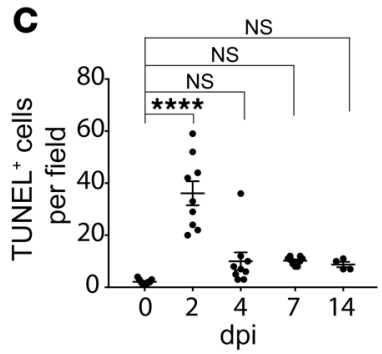

T1a/DAPI
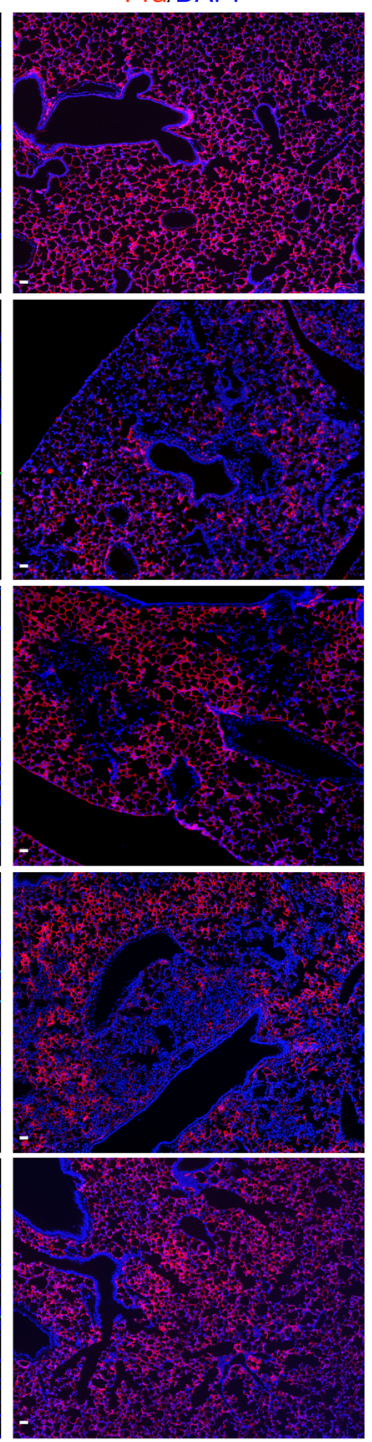

D

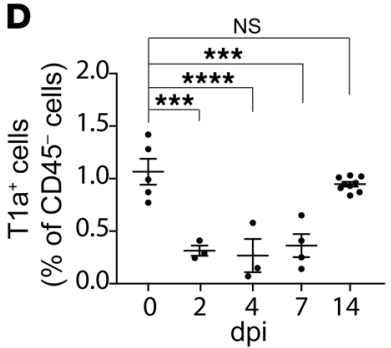

SPC/DAPI
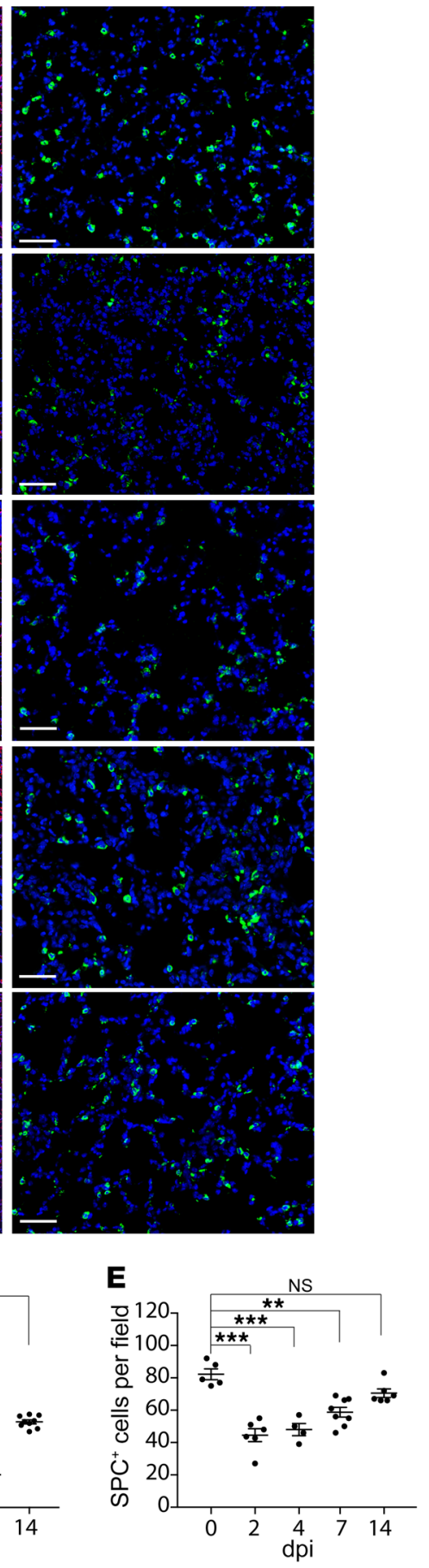

Figure 1. Alveolar epithelial injury and recovery in SpT4-infected mice. Lung tissues were collected at 0, 2, 4, 7, and 14 dpi with SpT4. (A) Immunostaining on lung sections with antibodies to the type 4 capsule of SpT4, T1a, and pro-SPC (SPC). Cell apoptosis was measured by TUNEL staining. Cell nuclei were stained with DAPI (blue). Scale bars: $50 \mu \mathrm{m}$. (B) Mouse lungs were homogenized, and lung lysates were plated for quantitative culture of colonizing pneumococci. (C) Quantification of cell apoptosis by counting TUNEL ${ }^{+}$cells on lung sections. (D) Lung cells were dissociated, and T1a ${ }^{+}$cells were quantified as percentage of total CD45- cells by flow cytometry. (E) Quantification of the number of SPC+ ${ }^{+}$cells on lung sections. $n \geq 10$ randomly selected fields per animal (C and E); $n=3-9$ per group (B-E). ${ }^{* *} P<0.01$; ${ }^{* *} P<0.001 ;{ }^{* * *} P<0.0001$, 1-way ANOVA.

dpi) (Supplemental Figure 3A). Inhibition of Hippo signaling leads to nuclear Yap/Taz localization and activity $(16,17)$. Nuclear Yap/ Taz expression was not detected in AECIIs of noninfected lungs (Figure 2F). However, we observed increases in nuclear Yap/Taz protein levels in AECIIs before (4 dpi) and during (7 dpi) differen- tiation toward AECIs (Figure 2F). Western blot confirmed higher levels of Yap/Taz protein in AECIIs purified from lungs at $7 \mathrm{dpi}$ compared with those from noninfected lungs (Figure $2 \mathrm{G}$ ). Moreover, nuclear locations of Yap/Taz proteins were also elevated in AECIIs at 7 dpi compared with noninfected AECIIs, as quantifica- 
A

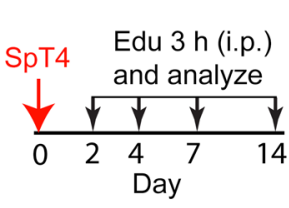

Edu SPC DAPI, 0 dpi

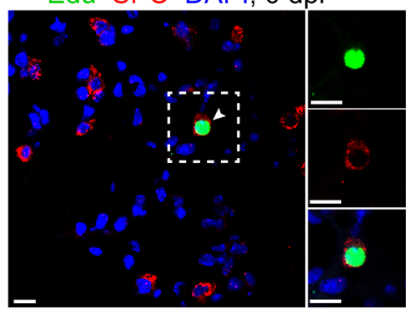

Edu SPC DAPI, 4 dpi

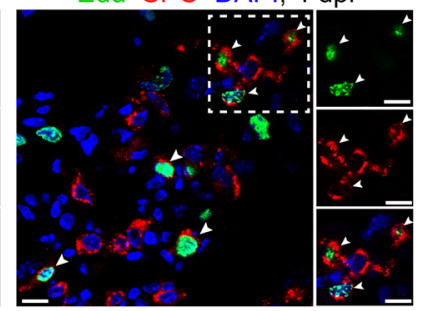

B

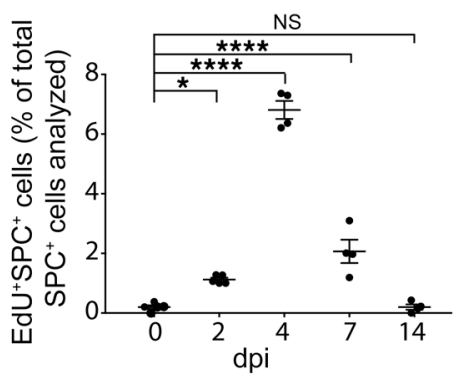

C GFP T1a DAPI, 0 dpi

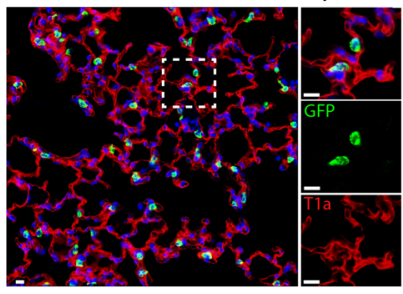

GFP T1a DAPI 7 dpi

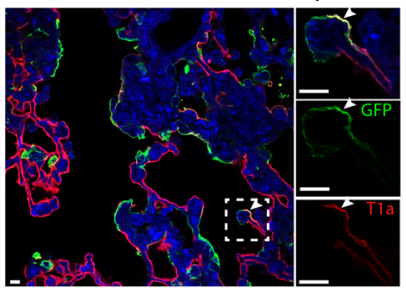

GFP T1a DAPI, 14 dpi

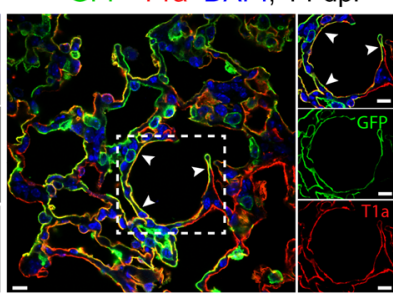

D

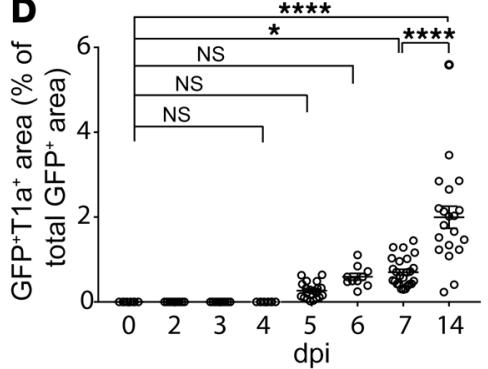

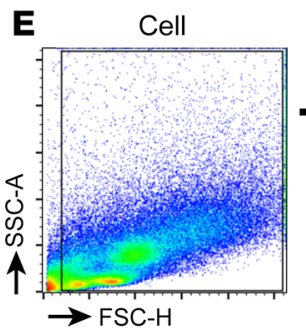
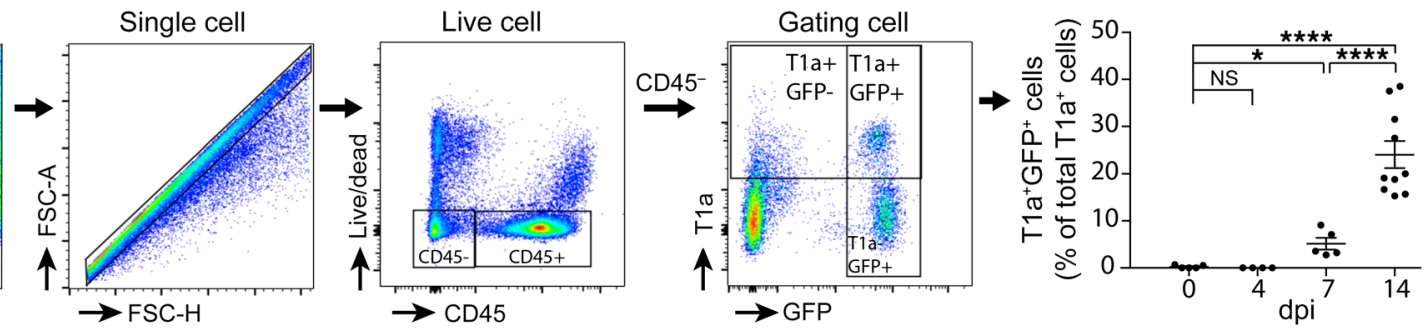

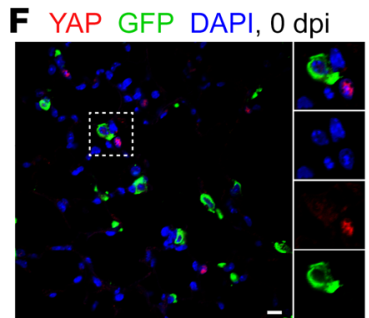

TAZ GFP DAPI, 0 dpi

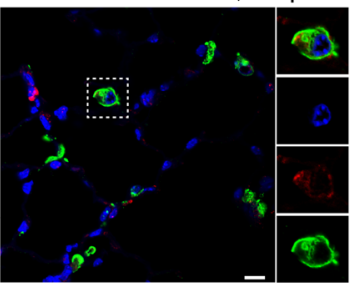

YAP GFP DAPI, 4 dpi

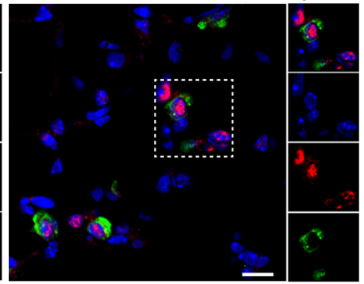

TAZ GFP DAPI, 4 dpi

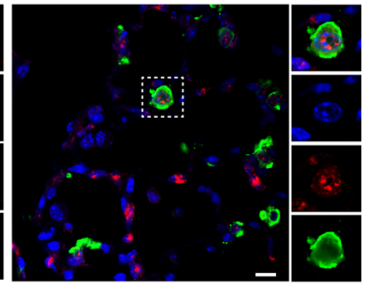

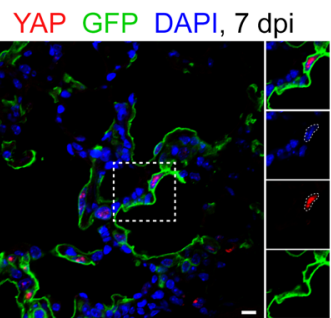

TAZ GFP DAPI, 7 dpi

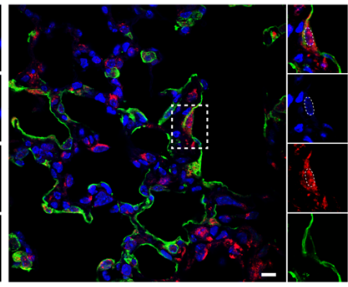

G
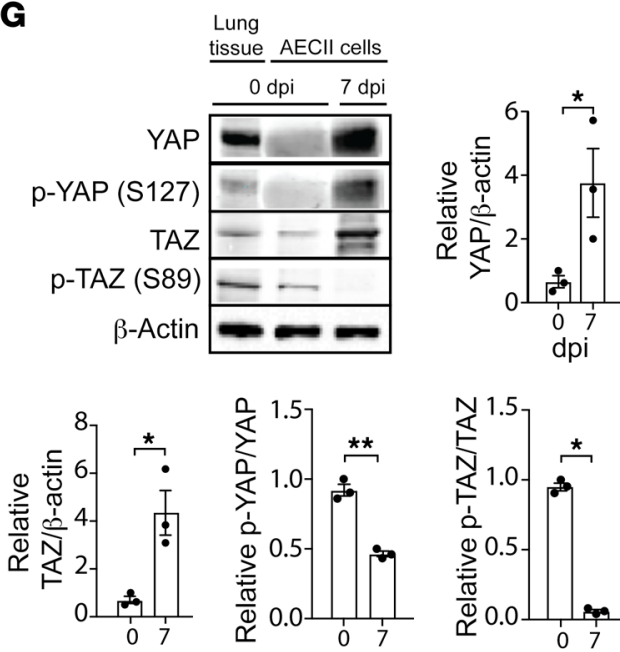

dpi

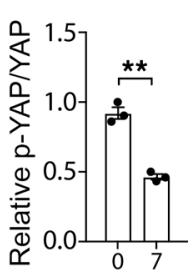

dpi

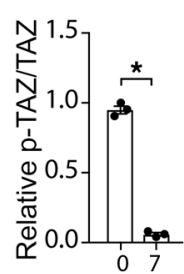

dpi

Figure 2. Alveolar epithelial regeneration and Yap/Taz expression in mouse lungs during bacterial pneumonia. (A) Schematic of experimental design (left) and confocal images of lung sections at $\mathrm{Odpi}$ and $4 \mathrm{dpi}$. AECIls in DNA synthesis phase were detected using Click-iT EdU Alexa Fluor (green) and coimmunostaining with antibody against pro-SPC (SPC) (red). Cell nuclei were stained with DAPI (blue). (B) Quantification of EdU $+5 P C^{+}$cells as percentage of total SPC+ cells analyzed ( 2200 SPC $^{+}$cells per animal). (C) Confocal images of lung sections of SPC-CreERT2, Rosa26-mTmG mice at 0, 7, and 14 dpi. Mice were administrated with 3 doses of tamoxifen to label SPC+ AEClls. Fourteen days after the last tamoxifen treatment, mice were infected with SpT4. AECII-to-AECI differentiation was visualized by coimmunostaining with antibodies against GFP (lineage-labeled AECIIs) and T1a (AECIs). Arrowheads point to regions double-positive for GFP and T1a. (D) Quantification of percentage of GFP+T1a area of total GFP+ area per field using Image). (E) Flow cytometry analysis of dissociated lung cells showing the percentage of GFP+T1a+ cells of total T1a+ cells at indicated time points. (F) Confocal images of lung sections of SPC-CreERT2, Rosa26-mTmG mice. Immunostaining with antibodies against GFP (lineage-labeled AECIIs) and Yap and Taz. Cell nuclei were stained with DAPI (blue). (C) Western blot using lung tissue lysates at 0 dpi or purified AEClls at 0 and $7 \mathrm{dpi}$, blotted with anti-YAP, anti-pYAP (Ser127), anti-Taz, anti-pTaz (S89), and anti- $\beta$-actin. Histograms showed average of total YAP or TAZ normalized to $\beta$-actin (loading control), together with average ratio of pYAP/YAP and pTAZ/TAZ. $n \geq 4$ per group (B, D, E); $n=3$ per group (G). ${ }^{*} P<0.05 ;{ }^{*} P<0.01$; ${ }^{* * * *} P<0.0001,1$-way ANOVA (B, D, E) and Student's $t$ test (G). Scale bars: $10 \mu \mathrm{m}$. 
tion of relative expression of phosphorylated Yap (p-Yap, Ser127) versus total Yap and p-Taz (Ser89) versus total Taz revealed lower levels of $\mathrm{p}$-Yap/Yap and $\mathrm{p}$-Taz/Taz in AECIIs at 7dpi than in noninfected AECIIs (Figure 2G).

Yap/Taz deletion in AECIIs impairs alveolar epithelial regeneration and causes prolonged fibrotic lesions during bacterial pneumonia. The nuclear localization of Yap and Taz in AECIIs in response to SpT4-induced lung injury could potentially augment alveolar regeneration by enhancing their targeting of regeneration-related genes. We tested this in the adult mouse lung with tamoxifeninducible Yap/Taz gene deletion in SPC ${ }^{+}$AECIIs. SPC-CreERT2, $\mathrm{Yap}^{\mathrm{fl} / \mathrm{fl}}$, Taz ${ }^{\mathrm{fl} / \mathrm{fl}}$, Rosa26-mTmG mice were administrated with tamoxifen i.p. to delete Yap and Taz in $\mathrm{SPC}^{+}$AECIIs (referred as Yap/Taz mutant mice). Yap/Taz deletion and reduced expression of their target genes were verified by qRT-PCR analysis performed on purified AECIIs 14 days after tamoxifen administration (Supplemental Figure 3B).

To gain insight into the role of Yap/Taz in AECIIs in the steadystate adult lung, we quantified the survival, self-renewal, and differentiation of lineage-labeled $\mathrm{SPC}^{+} \mathrm{AECIIs}$ at 9 days and 12 weeks after tamoxifen administration. We lineage labeled AECIIs by generating SPC-CreERT2, floxed Yap/Taz, Rosa26-mTmG mice so that a GFP reporter could be used to label and trace AECIIs in which Yap/Taz had been deleted. We found no detectable changes in $\mathrm{GFP}^{+}$cell apoptosis, as assessed by TUNEL staining at 9 days and 12 weeks after tamoxifen administration (Supplemental Figure 3, C and D). A transient decrease of DNA synthesis was observed in the lineage-labeled AECIIs from Yap/Taz mutant mice compared with SPC-CreERT2, Rosa26-mTmG control mice at 9 days after tamoxifen administration $(0.49 \% \pm 0.10 \%$ vs. $1.45 \% \pm 0.10 \%$; $P<0.001$; Supplemental Figure 3E). The decreased AECII cellcycle progression in Yap/Taz mutant mice was also observed by immunostaining of sectioned lungs for the cell cycle marker Ki67 (0.30\% $\pm 0.09 \%$ vs. $1.80 \% \pm 0.09 \%$; Yap/Taz mutant vs. SPC-CreERT2, Rosa26-mTmG control, respectively; $P<0.001$; Supplemental Figure 3F). By 12 weeks after tamoxifen administration, there were no significant differences in the percentage of lineage-labeled AECIIs that were $\mathrm{EdU}^{+}\left(\mathrm{EdU}^{+} \mathrm{GFP}^{+}\right)$between Yap/ Taz mutant and control mice $(0.16 \% \pm 0.02 \%$. vs $0.18 \% \pm 0.08 \%$; Supplemental Figure 3E). However, the level of $\mathrm{Ki}^{+} \mathrm{GFP}^{+}$was still lower in Yap/Taz mutant mice than that in control mice $(0.76 \% \pm$ $0.09 \%$ vs.1.44\% $\pm 0.4 \%, P<0.01$; Supplemental Figure 3F). Quantification of AECII-to-AECI differentiation by immunostaining and flow cytometry showed that Yap/Taz mutant mice exhibited reduced percentages of lineage-labeled AECIIs that had differentiated into AECIs $\left(\mathrm{GFP}^{+} \mathrm{T}^{+} \mathrm{a}^{+}\right)$at 12 weeks after tamoxifen administration compared with control mice $(1.68 \% \pm 0.25 \%$ vs. $2.70 \% \pm$ $0.10 \% ; P<0.05$; Supplemental Figure $3 G$ ). Histological analysis showed Yap/Taz mutant mice had normal alveolar structures at 4 weeks after tamoxifen administration, but exhibited patchy distribution of subtle interstitial infiltrates in the alveolar septa at 12 weeks after tamoxifen administration (Supplemental Figure 3H). FACS analysis revealed that Yap/Taz mutant lungs at 12 weeks after tamoxifen administration had increased numbers of inflammatory cells $\left(\mathrm{CD}^{\mathrm{H}} \mathrm{5}^{+}\right)$compared with SPC-CreERT2 control lungs (Supplemental Figure 3I). These data indicated that deletion of Yap/Taz in adult $\mathrm{SPC}^{+} \mathrm{AECIIs}$ led to decreased AECII proliferation and AECII-
to-AECI differentiation during steady-state tissue maintenance. Long-term deletion of Yap/Taz in $\mathrm{SPC}^{+}$AECIIs caused increased accumulation of inflammatory cells in the lung.

To determine whether Yap/Taz contributed to alveolar epithelial regeneration in vivo, Yap/Taz mutant mice were exposed to SpT4-induced lung injury. Yap/Taz mutant mice showed delayed recovery from bacterial pneumonia, as they regained body weight more slowly compared with the SPC-CreERT2 control mice (Supplemental Figure 4A). Yap/Taz mutant mice had higher levels of total protein in their bronchoalveolar lavage fluid (BALF) at $7 \mathrm{dpi}$ and 14 dpi compared with the control mice (Supplemental Figure $4 \mathrm{~B}$ ), indicating more damage to lung tissue integrity in Yap/Taz mutant mice. SPC-CreERT2 control mice started to regenerate AECIs $\left(\mathrm{T} 1 \mathrm{a}^{+}\right)$and AECIIs $\left(\mathrm{SPC}^{+}\right)$within a week after SpT4 infection and had full recovery of those cell populations by 14 dpi (Figure 1, $\mathrm{D}$ and $\mathrm{E}$ and Figure 3, A and B). In contrast, Yap/Taz mutant mice showed persistently low levels of AECIs and AECIIs for more than 2 weeks and only started to regenerate those cell populations by 21 dpi, indicating delayed alveolar epithelial regeneration following SpT4 infection (Figure 3, A and B). Yap/Taz mutant mice developed severe fibrotic lesions within the alveolar region at $14 \mathrm{dpi}$, as evidenced by increased Ashcroft scores in Trichrome-stained lung sections and collagen burden using hydroxyproline assay on lung tissue lysates compared with SPC-CreERT2 control mice (Figure $3, \mathrm{C}-\mathrm{E})$. Fibrotic lesions regressed over time, as seen by the gradual decreases in Ashcroft score and collagen burden by 56 dpi in Yap/Taz mutant lungs (Figure 3, D and E, and Supplemental Figure 4C). Lungs of single Yap mutant mice developed minor fibrotic lesions at $14 \mathrm{dpi}$, while lungs of single Taz mutant mice recovered from bacterial pneumonia without acquisition of fibrotic lesions at 14 dpi (Supplemental Figure 4D). We focused on Yap/Taz mutant mice in this study, since single mutant mice had minor or no phenotypes in response to SpT4 infection.

To determine the causes of impaired alveolar epithelial regeneration observed in Yap/Taz mutant mice, we profiled lung bacterial loads by measuring CFU and found no differences in bacteria number in lung homogenates and blood serum from Yap/Taz mutant mice compared with SPC-CreERT2 controls after SpT4 infection (Supplemental Figure 4, E and F). We next evaluated the effects of the Yap/Taz deletion on the process of alveolar epithelial damage. TUNEL staining of lung sections showed no differences in the number of apoptotic cells between Yap/Taz mutant and control lungs (Supplemental Figure 4, G-H). These results indicated that bacterial load and cell apoptosis were unlikely to explain the effects of the Yap/Taz deletion on alveolar epithelial regeneration and the development of lung fibrotic lesions during bacterial pneumonia.

To determine how Yap/Taz might contribute to alveolar epithelial regeneration, we examined their effects on AECII proliferation and AECII-to-AECI differentiation (Figure 4A). The proliferation index of AECIIs in Yap/Taz mutant mice at $7 \mathrm{dpi}$ was significantly lower than that in SPC-CreERT2, Rosa26mTmG control mice, as shown by the percentage of lineagelabeled AECIIs $\left(\mathrm{GFP}^{+}\right)$that were either EdU ${ }^{+}$or Ki67 $7^{+}$(Figure 4, B and C). At $14 \mathrm{dpi}, 24.1 \% \pm 2.1 \%$ of total lineage-labeled $\mathrm{GFP}^{+}$cells

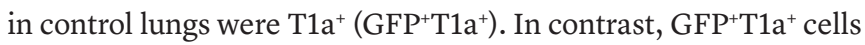
were reduced by $72.6 \%(6.6 \% \pm 2.1 \%)$ in Yap/Taz mutant lungs (Figure 4, D-F), indicating decreased AECII-to-AECI differentia- 
A

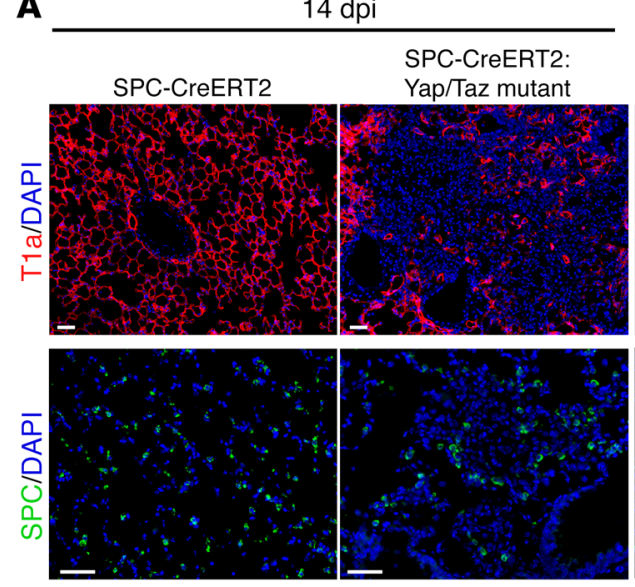

C

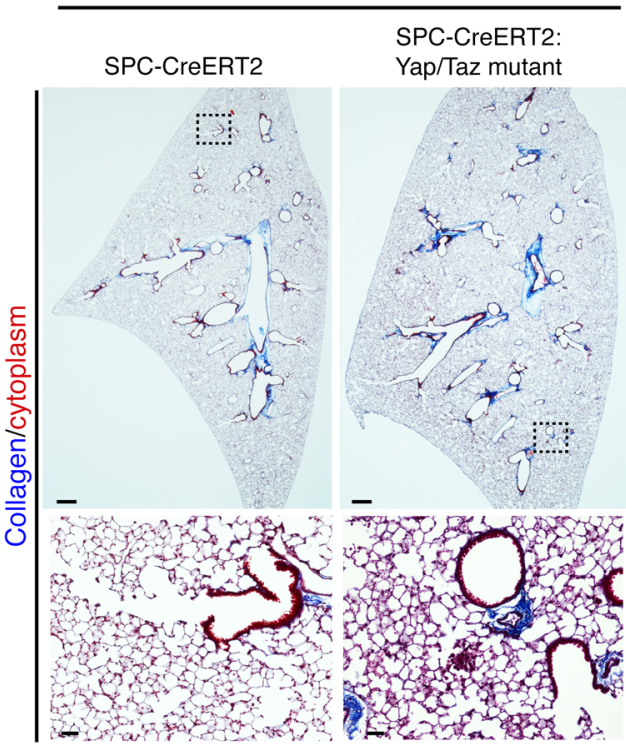

56 dpi
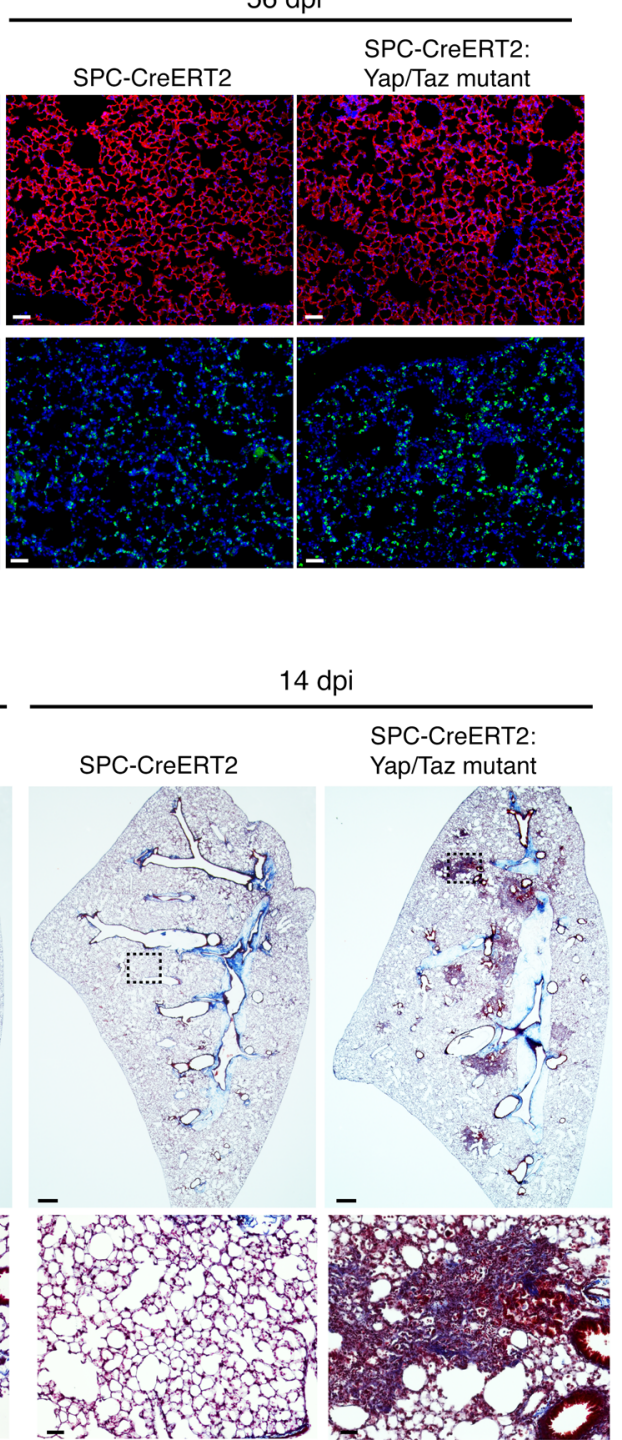

B
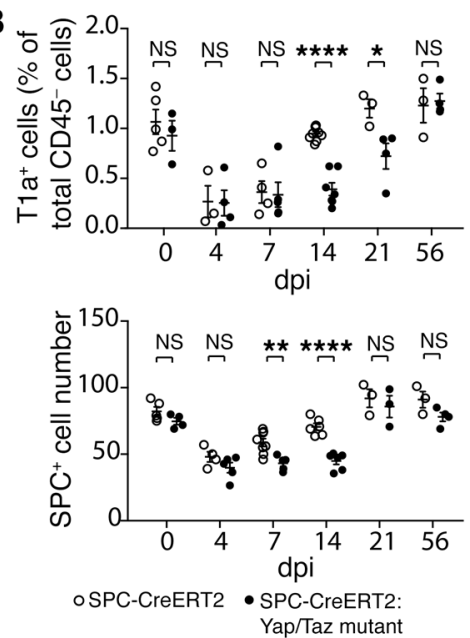

D

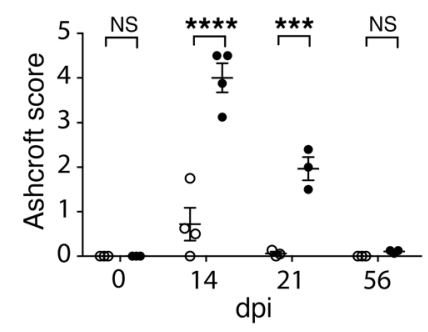

OSPC-CreERT2 • SPC-CreERT2: Yap/Taz mutant

E

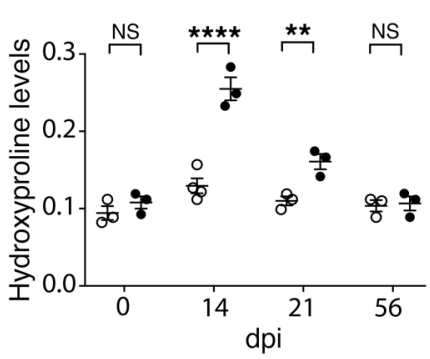

OSPC-CrEERT2 • SPC-CreERT2: Yap/Taz mutant

Figure 3. Phenotypes of Yap/Taz mutant lungs during bacterial pneumonia. (A) Immunostaining on lung sections with nuclei labeled by DAPI (blue) and antibodies to T1a (red) or pro-SPC (SPC) (green). (B) Lung cells were dissociated and T1a+ cells were quantified as percentage of total CD45- cells by flow cytometry. SPC cells were quantified by counting the number of SPC cells per field ( $\geq 10$ randomly selected fields per animal) ( $n=3-8$ per group). (C) Lung tissue sections were stained with Alcian blue and Nuclear Fast Red. Lung fibrotic lesions were quantified by measuring (D) Aschcroft score and (E) hydroxyproline assay ( $n=3-4$ per group). ${ }^{*} P<0.05 ;{ }^{* *} P<0.01$; ${ }^{* *} P<0.001$; ${ }^{* * *} P<0.0001,2$-way ANOVA. Scale bars: $50 \mu \mathrm{m}$ (A, C [bottom panel]); $500 \mu \mathrm{m}$ (C [top panel]).

tion in Yap/Taz mutant lungs. The AECII-to-AECI differentiation defect was persistent in Yap/Taz mutant lungs, as the levels of AECIs derived from lineage-labeled AECIIs $\left(\mathrm{GFP}^{+} \mathrm{T}^{+} \mathrm{a}^{+}\right)$were still significantly lower in Yap/Taz mutant lungs at 56 dpi than in SPCCreERT2, Rosa26-mTmG control lungs (Figure 4F). Collectively, these data indicated that loss of Yap/Taz in AECIIs diminished alveolar epithelial regeneration derived from preexisting SPCexpressing AECIIs and led to prolonged fibrotic lesions in the lung during bacterial pneumonia.

Yap/Taz regulate AECII inflammatory responses through targeting I $\mathrm{B} a$. To understand the underlying mechanisms for these observations, we performed microarray analysis on lineagelabeled AECIIs $\left(\mathrm{GFP}^{+}\right)$isolated from Yap/Taz mutant and SPC-
CreERT2 control lungs at $8 \mathrm{dpi}$. After normalization of data sets, we used ToppGene to identify pathways that were induced or repressed (FDR, $<5 \%$ and $>2$-fold expression) in $\mathrm{GFP}^{+}$AECIIs of Yap/Taz mutant mice. While gene ontologies associated with negative regulation of the apoptotic process, positive regulation of cell proliferation, and differentiation were repressed in Yap/ Taz mutant mice, gene ontologies associated with negative regulation of cell differentiation, inflammation response, leukocyte chemotaxis, and lipid metabolic process were all induced in Yap/Taz mutant mice (Supplemental Figure 5A and Supplemental Tables 1 and 2). qRT-PCR confirmed decreased expression of Yap, Taz, tand Yap/Taz-dependent target genes, including

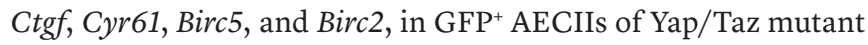


A
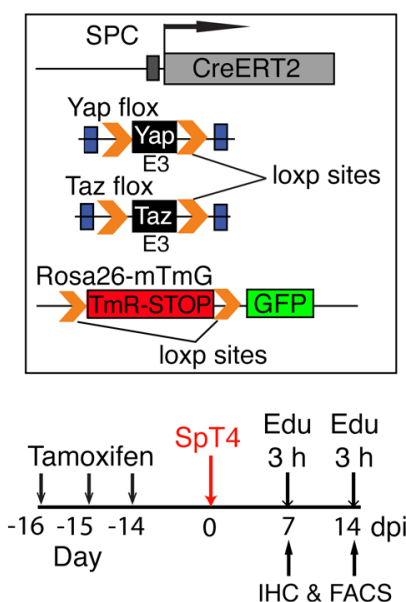

B
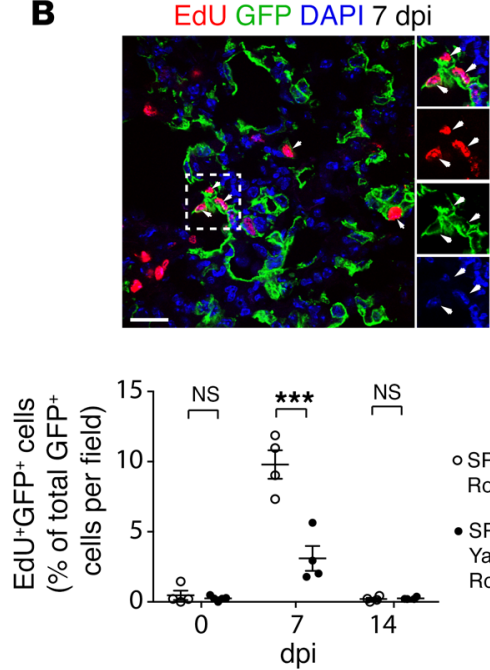

C
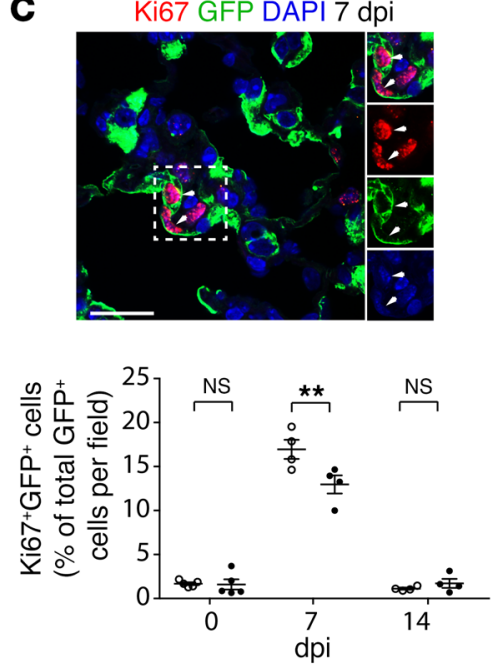
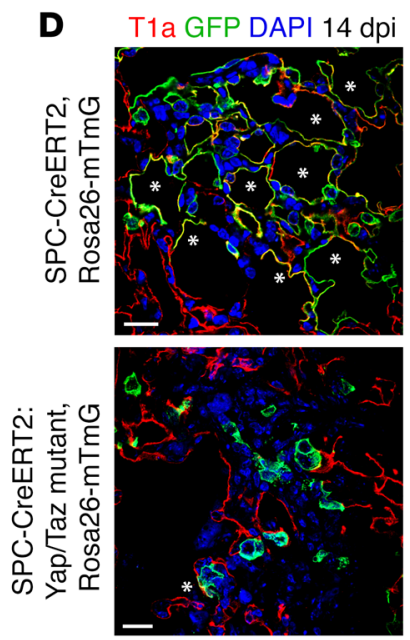

GFP
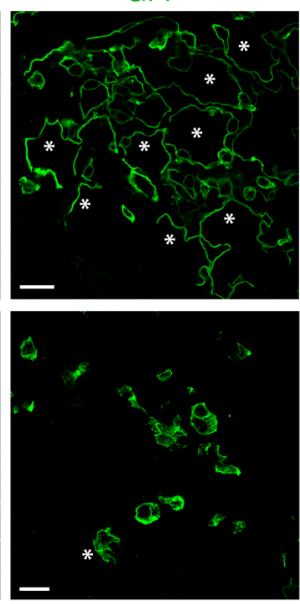

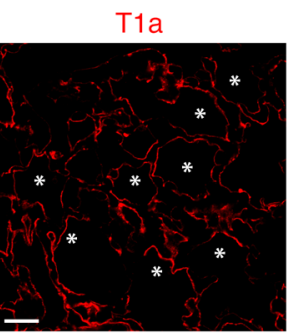

- SPC-CreERT2,

Rosa26-mTmG

- SPC-CreERT2:

Yap/Taz mutant Rosa26-mTmG

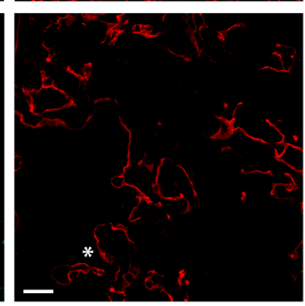

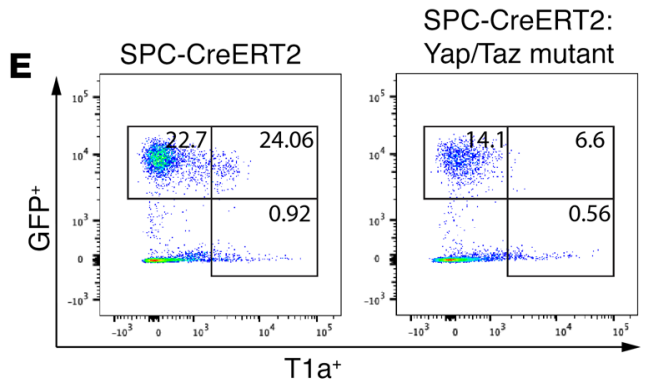

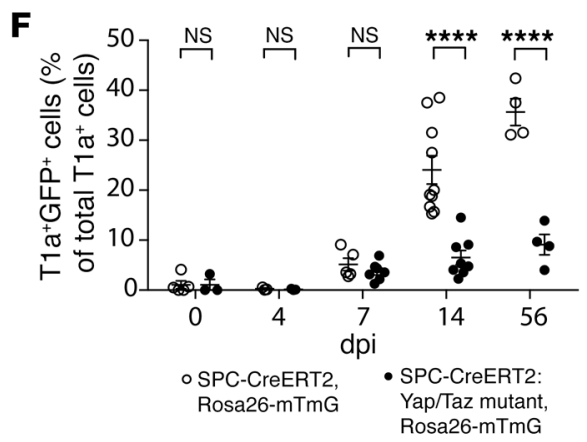

Figure 4. AECII proliferation and differentiation in Yap/Taz mutant lungs during bacterial pneumonia. (A) Schematic of experimental design for studies shown in B-F. (B) Lung tissue sections from SPC-CreERT2, Rosa26-mTmG mouse were immunostained with DAPI (blue) and antibody against GFP (lineagelabeled AECIIs) (green), and colabeling with Click-iT EdU Alexa Fluor (red) and confocal images were taken. The percentages of GFP+EdU' cells of total GFP+ cells per field were graphed (bottom panel). (C) Confocal image of lung section from SPC-CreERT2, Rosa26-mTmG mouse at 7 dpi with nuclei labeled by DAPI (blue) and antibodies against GFP (green) and Ki67 (red). Percentages of GFP+Ki67+ cells of total GFP+ cells per field were graphed (bottom panel). (D) Confocal images of lung section at 14 dpi with nuclei labeled by DAPI (blue) and antibodies against GFP (green) and T1a (red). Asterisks indicate regions double-positive for GFP and T1a. (E) Lung cells were dissociated and flow cytometry was performed by gating on GFP+T1a+. The numbers in the top left gates represent all GFP+ cells of total live CD45- cells. The numbers in the top right gates represent GFP+ $\mathrm{Tla}^{+}$cells of total $\mathrm{T1}^{+}$cells. The numbers in the bottom right gates represent

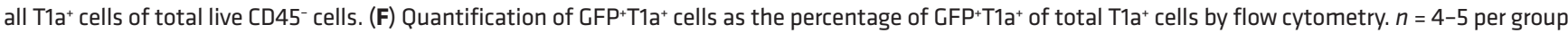
(B and C); $n=4-8$ per group (F). ${ }^{* *} P<0.01 ;{ }^{* *} P<0.001 ;{ }^{* * *} P<0.0001,2$-way ANOVA. Scale bars: $25 \mu \mathrm{m}$ (B and C); $20 \mu \mathrm{m}$ (D).

mice at 7 dpi compared with those of SPC-CreERT2 control mice (Figure 5A). The expression of several markers associated with positive regulation of cell proliferation and differentiation, including Fgf1, Fgfr3, Tgfb2, Wnt3a, Bmp4, and Egfr, was significantly reduced in $\mathrm{GFP}^{+}$AECIIs of Yap/Taz mutant mice (Figure $5 \mathrm{~A})$. These data suggested that loss of Yap/Taz in AECIIs led to decreased proliferation and differentiation of AECIIs in response to SpT4-induced lung injury. Notably, while expression of $N f k b 1$ itself was unchanged, expression of $N f k b 1$ repressor genes $I k b a$
(Nfkbia) and Ikbb (Nfkbib) was significantly decreased in Yap/Taz mutant $\mathrm{GFP}^{+}$AECIIs (Figure 5A). In contrast, the expression of NF-kB-dependent target genes, including Cxcl3 and Ccl21a, was significantly increased in Yap/Taz mutant $\mathrm{GFP}^{+}$AECIIs (Figure $5 \mathrm{~A})$. We detected a significant increase in IL-1b protein, a cytokine known to expand differentiated T cells (18), in BALF from Yap/Taz mutant mice at both $7 \mathrm{dpi}$ and $14 \mathrm{dpi}$ compared with that from SPC-CreERT2 control mice (Figure 5B). Cytokine array on lung tissue lysates showed the higher levels of cytokines, includ- 

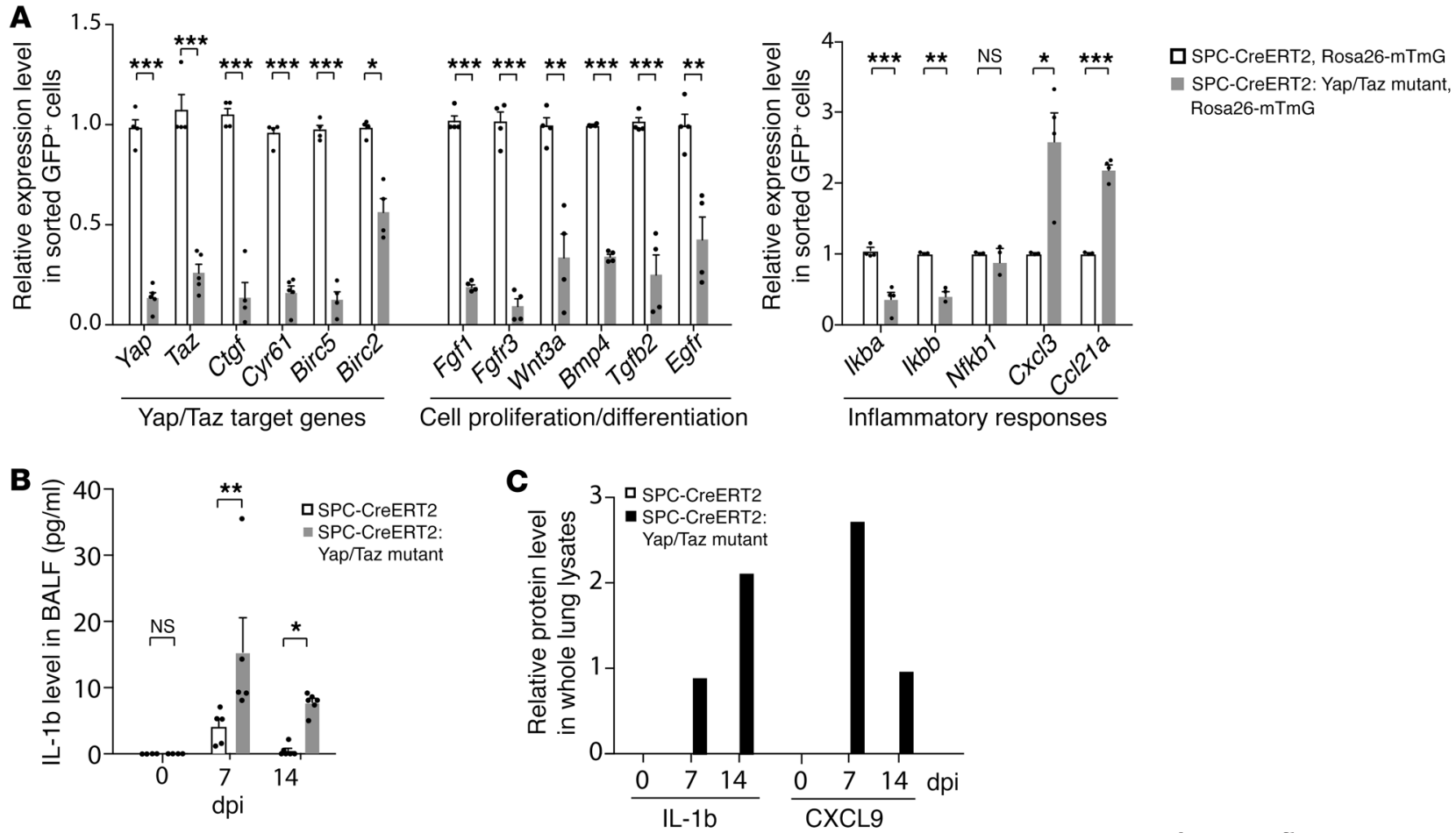

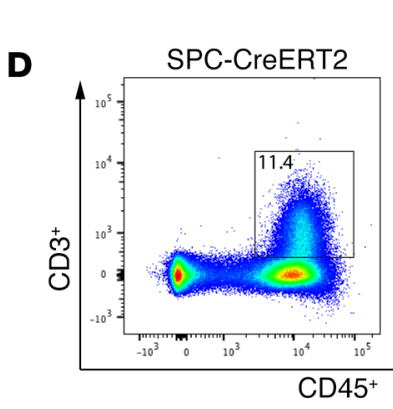

SPC-CreERT2:

Yap/Taz mutant

\section{$\mathbf{E}$}

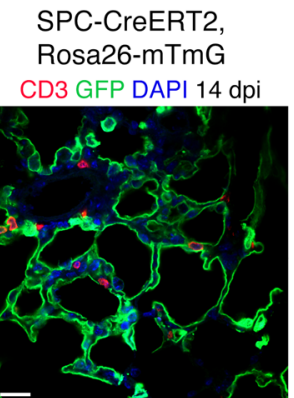

SPC-CreERT2,

\section{SPC-CreERT2:}

Yap/Taz mutant,

Rosa26-mTmG
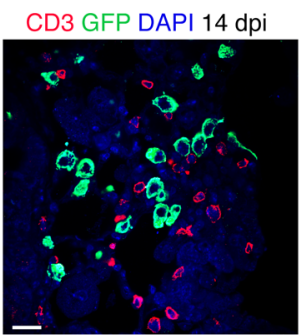

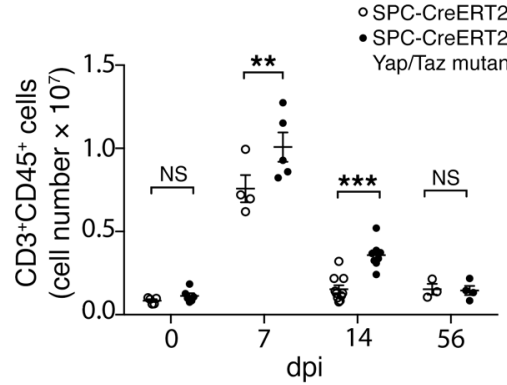

$\mathbf{F}$

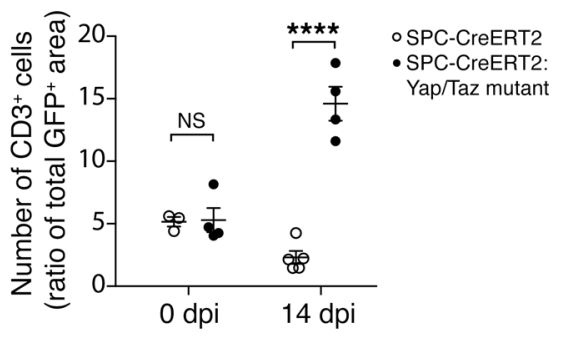

Figure 5. Inflammatory responses in Yap/Taz mutant lungs. (A) GFP ${ }^{+}$ AECIls at $7 \mathrm{dpi}$ were sorted by FACS and analyzed by qRT-PCR ( $n=4$ per group). (B) BALF was analyzed for IL-1b by ELISA assay ( $n=4-6$ per group). (C) Cytokine assay showed protein levels of IL-1b and CXCL9 in mouse lung lysates ( $n=1$ per group). (D) Flow cytometry of dissociated lung cells was performed by gating on $\mathrm{CD}^{+} \mathrm{CD}_{45} 5^{+}$cells, and quantification of total number of $\mathrm{CD}^{+} \mathrm{CD}^{+} 5^{+}$cells in the lung was graphed ( $n=3-10$ per group). (E) Confocal images of lung sections at $14 \mathrm{dpi}$ with nuclei labeled by DAPI (blue) and antibodies to GFP (green) and CD3 (red). Scale bars: $20 \mu \mathrm{m}$. (F) Quantification of the number of $\mathrm{CDB}^{+}$ cells as the ratio of $\mathrm{CD3}^{+}$cell number versus $\mathrm{GFP}^{+}$area per field using Imagel ( $n=3-5$ per group). ${ }^{*} P<0.05$; ${ }^{* *} P<0.01$; ${ }^{* * *} P<0.001$; ${ }^{* * * *} P<0.0001$, Student's $t$ test (A) and 2-way ANOVA (B, D, F). ing IL-1b as well as CXCL9, a T cell chemotactic cytokine (19), in Yap/Taz mutant mice at $7 \mathrm{dpi}$ and 14 dpi compared with SPCCreERT2 control lungs (Figure 5C and Supplemental Figure 5, $\mathrm{B}$ and $\mathrm{C})$. Consistent with the elevated expression of genes and cytokines associated with $\mathrm{T}$ cell inflammatory responses in Yap/ Taz mutant AECIIs, we observed a significant increase in the number of $\mathrm{CD}^{+} \mathrm{T}$ cells in Yap/Taz mutant lungs at $7 \mathrm{dpi}$ and 14 dpi (Figure 5D). Immunostaining on sectioned lung tissues confirmed the increased number of $\mathrm{CD}^{+} \mathrm{T}$ cells located in lung alveoli of Yap/Taz mutant mice at 14 dpi (Figure 5, E and F). Interestingly, $\mathrm{CD}^{+} \mathrm{T}$ cells were enriched in areas where AECIIs failed to differentiate into AECIs in Yap/Taz mutant lungs (Figure 5F). In addition, the number of macrophages $\left(\mathrm{CD} 11 \mathrm{c}^{+} \mathrm{CD} 64^{+}\right)$in Yap/Taz mutant lungs was lower at $7 \mathrm{dpi}$, but higher at $14 \mathrm{dpi}$, than those in SPC-CreERT2 control lungs (Supplemental Figure 5D). In contrast, the number of neutrophils $\left(\mathrm{Ly} 6 \mathrm{G}^{+}\right.$) was not significantly changed in Yap/Taz mutant lungs compared with control lungs (Supplemental Figure 5D). The effect of Yap/Taz mutant AECIIs on lung inflammation waned over time, as seen by the equivalent levels of $\mathrm{T}$ cells $\left(\mathrm{CD}^{+}\right)$and macrophages $\left(\mathrm{CD} 11 \mathrm{c}^{+} \mathrm{CD} 64^{+}\right)$ at 56 dpi between Yap/Taz mutant and SPC-CreERT2 controls (Figure 5D and Supplemental Figure 5D). These results indicated that loss of Yap/Taz in AECIIs led to prolonged inflammatory responses in lung alveoli during bacterial pneumonia. 
Because Yap/Taz mutant AECIIs had reduced expression of $I k b a$ and $I k b b$, we hypothesized that an I $\mathrm{B} / \mathrm{NF}-\kappa \mathrm{B}$ pathway drives the inflammatory responses in Yap/Taz mutant lungs during bacterial pneumonia. Given recent identification of the Yap/IкB signaling axis as a potential means of generating inflammatory response in Drosophila post-Gram ${ }^{+}$bacterial infection (20), we investigated whether Yap/Taz controls IкB levels in the mouse lung. Examination of the Ikba genomic locus revealed 5 potential Tead-binding motifs (TBM) within a $10 \mathrm{~kb}$ region upstream of the transcriptional start site (Figure 6A), while there was no potential TBM in the 10 $\mathrm{kb}$ region upstream of the $I k b b$ genomic locus. For the subsequent experiments, we focused on I $\mathrm{B}$ a because it was shown to be the most efficient I $\mathrm{B}$ molecule to control NF- $\kappa$ B transcriptional activity in previous studies (21-23). We first performed ChIP assays on chromatin obtained from the murine lung epithelial cell line MLE15 using either an anti-Tead or anti-Yap antibody to determine whether Tead and Yap were associated with any of the 5 TBMs. ChIP analysis showed that Yap and Tead were associated with the TBM1 site of Ikba (Figure 6B). To determine whether the TBM1 site was responsive to Tead transactivation, MLE-15 cells were transfected with the pGL3 luciferase reporter containing TBM1-3 region along with an expression plasmid encoding murine Tead2 (Figure 6C). This study showed that expression of Tead 2 transactivated the pGL3-IкBa-TBM1-3.luc reporter. Coexpression of Tead2 with Yap or Taz further enhanced the transactivation of the pGL3IкBa-TBM1-3.luc reporter (Figure 6C). In contrast, expression of Tead2 did not activate the pGL3-IкBa-TBM1-3 mutant.luc reporter in which TBM1 was mutated (Figure 6C).

IкBa has previously been shown to be required for inhibiting $\mathrm{NF}-\kappa \mathrm{B}$-mediated inflammatory response in the lung (24), raising the possibility that the regulation of IкBa expression by Yap/Taz may affect NF- $\kappa \mathrm{B}$ transcriptional activity. To test this hypothesis, AECIIs from adult mouse lungs were cultured for 48 hours in the presence of a Yap shRNA lentivirus that inhibited Yap expression (25). Gene expression analysis showed that expression of Yap shRNA reduced the expression of Ikba in AECIIs compared with scrambled shRNA lentivirus-treated AECIIs (Supplemental Figure 5E). Analysis of NF- $\kappa B$ transcriptional activity showed that Yap shRNA-treated AECIIs had higher levels of NF- $\mathrm{B}$ transcriptional activity compared with scrambled shRNA-treated or nontreated control AECIIs (Figure 6D). Moreover, AECIIs purified from Yap/Taz mutant mice at $7 \mathrm{dpi}$ showed higher levels of NF- $\mathrm{KB}$ transcriptional activities than those from SPC-CreERT2 control mice (Figure 6E). In contrast, overexpression of Yap or Taz in AECIIs using YAP-5SA or TAZ-S89A adeno-associated virus type 6 (AAV6) led to increased Ikba expression and decreased NF- $\kappa \mathrm{B}$ transcriptional activity compared with AAV6 GFP-treated AECIIs (Supplemental Figure 5, E and F). To determine whether Yap/Taz-induced IкBa expression affected NF- $\kappa \mathrm{B}$ signaling, MLE-15 cells were transfected with the $\mathrm{NF}-\kappa \mathrm{B}$ response luciferase reporter. This study showed that expression of Yap shRNA led to increased transactivation of the NF-кB response luciferase reporter. In contrast, overexpression of Yap or Taz reduced the transactivation of the NF- $\kappa \mathrm{B}$ response luciferase reporter (Figure 6F). These results indicated that Yap/Taz regulated $\mathrm{NF}-\kappa \mathrm{B}$ response through targeting I $\mathrm{Ba}$ in AECIIs (Figure 6G).

$I \kappa B a$ overexpression promotes inflammatory resolution and alveolar epithelial regeneration in Yap/Taz mutant lung. To determine whether loss of IкBa expression was responsible for the prolonged inflammatory response and delayed alveolar epithelial regeneration observed in Yap/Taz mutant mice, we restored IкBa expression in Yap/Taz mutant lungs using the AAV6 vector for the expression of murine IкBa. We first evaluated whether intratracheal instillation of AAV6 led to efficient gene transfer and expression in AECIIs. Mouse lungs treated with AAV6-GFP at a dose of $2.5 \times 10^{10} \mathrm{vp} /$ animal exhibited abundant GFP staining in the lung alveolar region and much lower staining in the airway epithelium at 48 hours after instillation (Supplemental Figure 6A). In lung alveoli, 97.3\% $\pm 1.1 \%$ of $\mathrm{GFP}^{+}$cells were $\mathrm{SPC}^{+}$and less than $2.5 \%$ of $\mathrm{GFP}^{+}$cells were $\mathrm{CD}^{+}$ (Supplemental Figure 6B). These results indicated that intratracheal instillation of AAV6 resulted in preferential transduction of $\mathrm{SPC}^{+}$ AECIIs in mouse lungs. Next, Yap/Taz mutant mice were administrated with AAV6-IкBa at a dose of $2.5 \times 10^{10} \mathrm{vp} /$ animal through intratracheal instillation at $7 \mathrm{dpi}$. Twenty-four hours later, AECIIs were purified and performed for gene expression analysis by qRT-PCR. I $\mathrm{BBa}$ overexpression in Yap/Taz mutant lungs restored $I k B a$ transcription, while $I k B a$ expression in inflammatory cells $\left(\mathrm{CD} 45^{+}\right)$was unchanged (Supplemental Figure 6C). Restoration of $I k B a$ expression in Yap/Taz mutant lungs was sufficient to inhibit NF- $\kappa \mathrm{B}-$ mediated gene expression in AECIIs, since AAV6-IкBatreated AECIIs exhibited decreased expression of NF- $\mathrm{BB}-$ dependent genes ( $\mathrm{Cxcl3}$, Ccl21a) compared with PBS treatment (Supplemental Figure 6C). We observed a significant decrease in IL-1b protein in BALF from AAV6-IкBa-treated Yap/Taz mutant mice at 14 dpi (Figure 7, A and B). Cytokine array showed that AAV6IкBa-treated Yap/Taz mutant lungs at 14 dpi produced lower levels of cytokines, including cytokines associated with $\mathrm{T}$ cell accumulation (IL-1b, CXCL9) and antiinflammatory resolution responses (TIMP-1, TREM-1) (Figure 7C and Supplemental Figure 7, A and B). PBS-treated Yap/Taz mutant lungs showed results similar to those of AAV6-null-treated Yap/Taz mutant lungs and were used as the controls in this study (Supplemental Figure 7, A-C). The impairment in inflammatory resolution in Yap/Taz mutant lung was

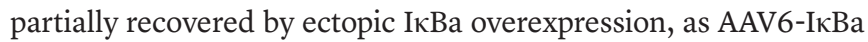
treatment on Yap/Taz mutant mice led to a significant reduction in the number of $\mathrm{CD}^{+} \mathrm{T}$ cells in the lung at $14 \mathrm{dpi}$ (Figure 7D). Inflammatory responses were not completely absent in AAV6IкBa-treated Yap/Taz mutant lungs, however, as the numbers of neutrophils $\left(\mathrm{Ly}_{6 \mathrm{G}}{ }^{+}\right)$and macrophages $\left(\mathrm{CD} 11 \mathrm{c}^{+} \mathrm{CD} 64^{+}\right)$were equivalent between AAV6-IкBa-, PBS-, and AAV6-null-treated Yap/Taz mutant lungs (Supplemental Figure 7, D and E).

We next assessed whether partial inflammatory resolution was sufficient to accelerate alveolar epithelial regeneration and regression of fibrotic lesions in Yap/Taz mutants. Analyses of lung sections by immunostaining and flow cytometry showed AAV6-IкBa-treated Yap/Taz mutant lungs exhibited significant improvement in the recovery of AECIs at 14 dpi compared with PBS- or AAV6-null-treated Yap/Taz mutant lungs (Figure 7, E-G, and Supplemental Figure 7C). Moreover, AAV6-IкBa treatment led to improved lung tissue integrity in Yap/Taz mutant mice, as gauged by the total protein measurement in their BALF (Figure $7 \mathrm{H}$ and Supplemental Figure 7C). The regenerated AECIs in AAV6IкBa-treated Yap/Taz mutant lungs were not associated with the differentiation of preexisting $\mathrm{SPC}^{+} \mathrm{AECIIs} \mathrm{into} \mathrm{AECIs,} \mathrm{since}$

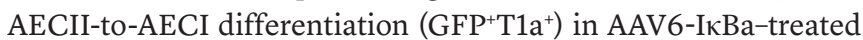


A

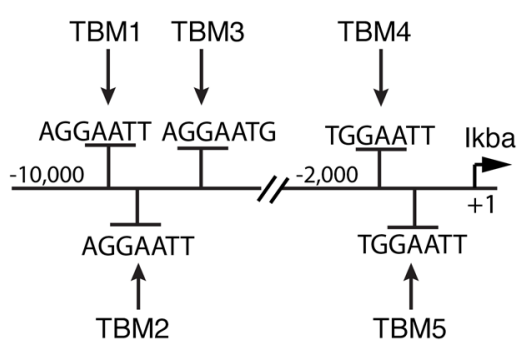

TEAD binding sequence: WGGAATKH

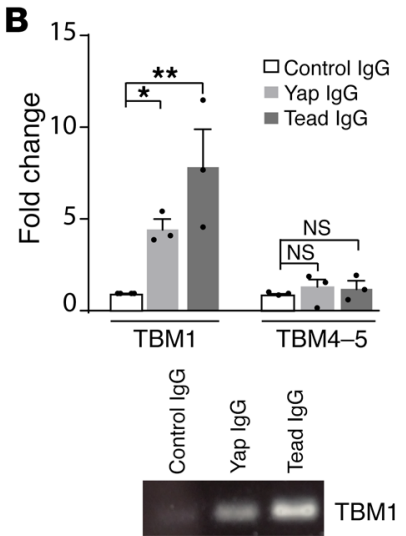

C pGL3-Ikba-TBM1-3.Iuc: TBM1 TВM2 TBM3

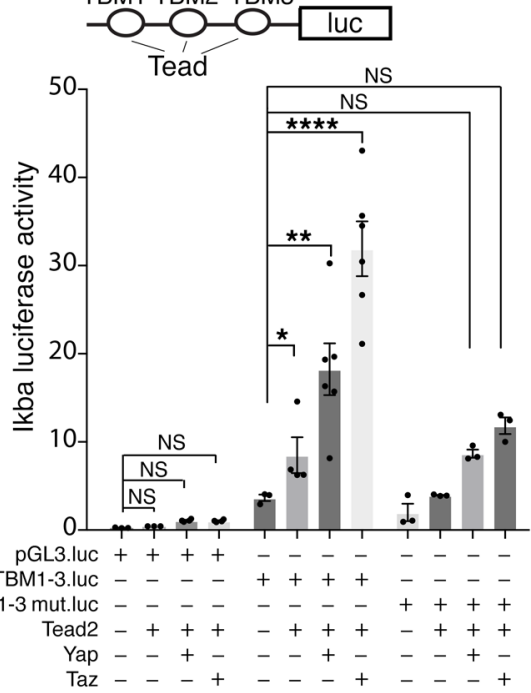

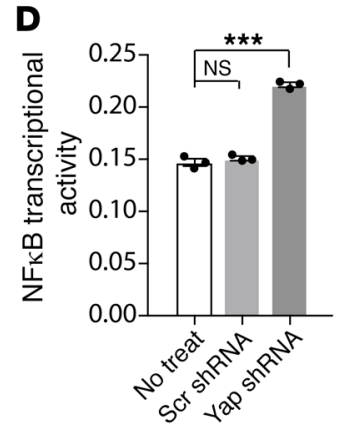
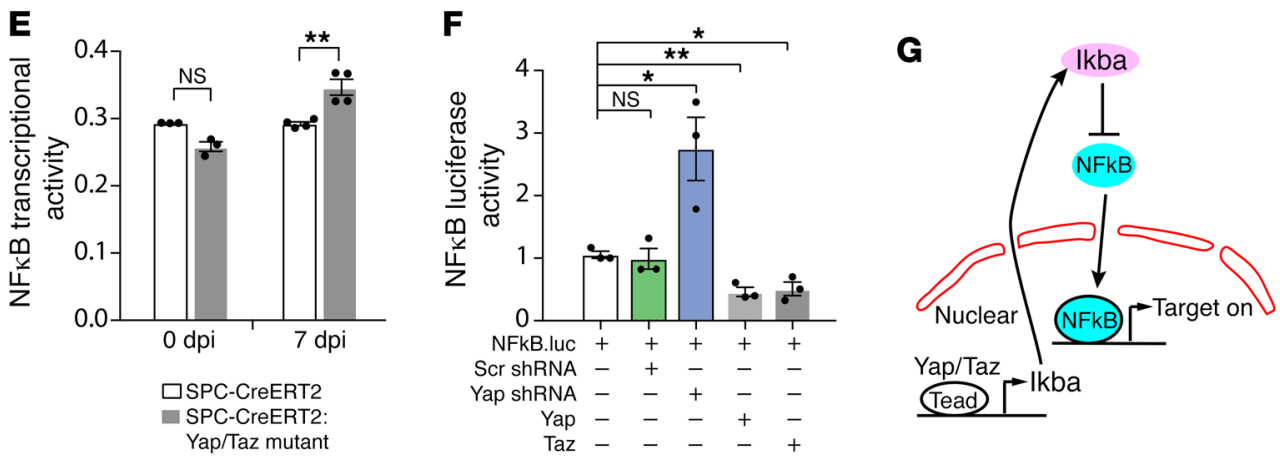

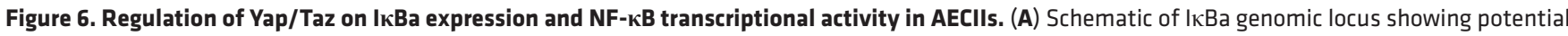
Tead binding sites (TBM) in the $10 \mathrm{~kb}$ upstream of the IкBa transcription start site. (B) Chromatin from MLE-15 cells was immunoprecipitated with either Tead or Yap antibody, and qRT-PCR results were graphed $(n=3)$. (C) MLE-15 cells were transfected with pGL3 vector containing a IאBa-TBM1-3 or a IאBaTBM1-3 mutation, in which TBM1 was mutated, along with the expression plasmid encoding either murine Tead2 or Yap or Taz. Twenty-four hours after transfection, cells were processed for luciferase activity measurement ( $n=3-6$ per group). (D) Adult WT mouse AECIIs were purified, cultured, and infected with either Yap shRNA lentivirus or scramble shRNA lentivirus or were given no treatment. Forty-eight hours after lentiviral infection, cells were processed for NF- $\mathrm{KB}$ transcription activity measurement. (E) AEClls were purified from mouse lungs at $0 \mathrm{dpi}$ and $7 \mathrm{dpi}$, and their NF- $\mathrm{BB}$ transcriptional activity was graphed. (F) MLE-15 cells were infected with either Yap shRNA lentivirus or scramble shRNA lentivirus or were given no treatment. Forty-eight hours after lentiviral infection, cells were transfected with NF- $\kappa B$ luciferase vector containing NF- $\kappa B$ response elements, along with an expression plasmid encoding either murine Yap or Taz. Twenty-four hours after transfection, cells were processed for luciferase activity measurement $(n=3)$. (C) Schematic model of

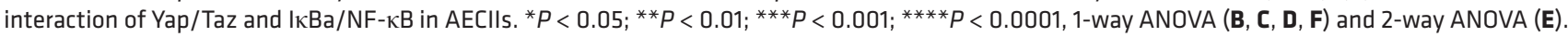

Yap/Taz mutant lungs was equivalent to that in PBS-treated Yap/Taz mutant lungs (Supplemental Figure 8, A-C), suggesting the recovered AECIs were contributed by non-lineage-labeled AECIIs. We measured Ashcroft scores in Trichrome-stained lung sections and collagen burden in lung tissue lysates at $14 \mathrm{dpi}$ and found a significant decrease in lung fibrotic lesions in AAV6IкBa-treated Yap/Taz mutant mice compared with PBS- or AAV6null-treated mice (Figure 7, I-K, and Supplemental Figure 7C). Together, these findings indicated that restoration of IкBa expression in Yap/Taz mutant lung promoted inflammatory resolution and accelerated alveolar epithelial regeneration and regression of lung fibrotic lesions during bacterial pneumonia.

\section{Discussion}

Bacterial pneumonia has long been associated with lung damage, but how the host recovers from lung damage and the potential role of lung regeneration versus fibrosis have not been fully understood. Here, we investigated the recovery from bacterial pneumonia in mice infected by $S$. pneumoniae. We found that mice had massive tissue damage exclusively in lung alveoli during bacterial pneumonia. Mice recovering from pneumococcal infection lacked detectable lung fibrosis, suggesting that considerable regeneration of lung tissue must be acting during this recovery. Numerous studies have demonstrated alveolar epithelial regeneration after injury involving $\mathrm{SPC}^{+} \mathrm{AECIIs} \mathrm{(3).} \mathrm{We} \mathrm{showed} \mathrm{that} \mathrm{SPC}^{+} \mathrm{AECIIs}$ increased proliferation within 7 dpi. We also showed that lineagelabeled SPC ${ }^{+} \mathrm{AECIIs} \mathrm{differentiated} \mathrm{to} \mathrm{AECIs} \mathrm{at} \mathrm{sites} \mathrm{of} \mathrm{damaged}$ lung alveoli during 7-14 dpi, coinciding with the time frame when inflammation was resolved in the lung.

AECIIs synthesize and secrete surfactant and proliferate and differentiate into AECIs after injury in order to maintain the integrity of the alveolar wall. AECIIs also secrete cytokines and 
A

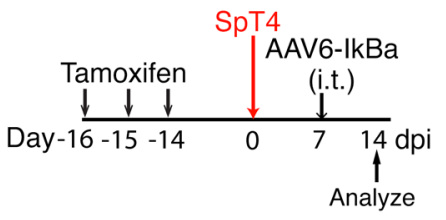

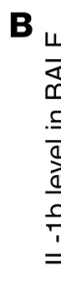

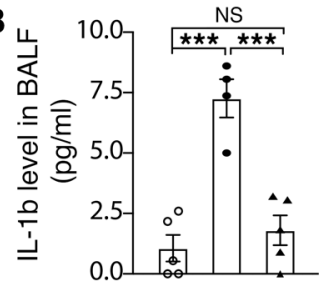

C

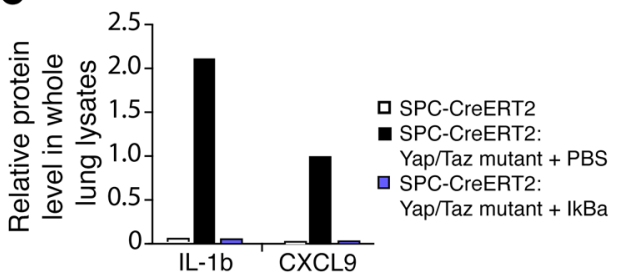

Yap/Taz mutant + PBS

SPC-CreERT2:

IL-1b CXCL9

D

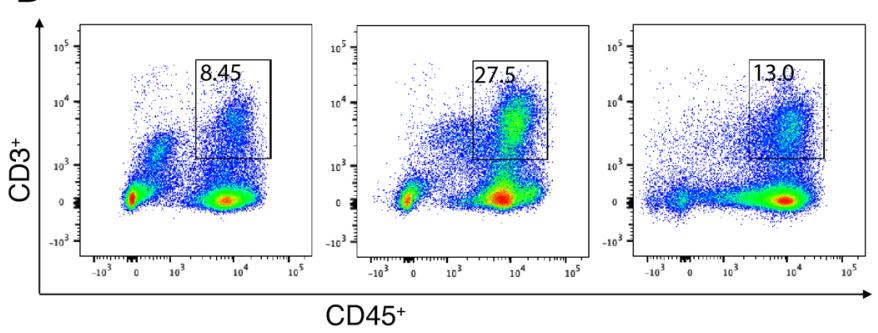

SPC-CreERT

Yap/Taz mutant + PBS

- SPC-CreERT2:

Yap/Taz mutant + lkBa

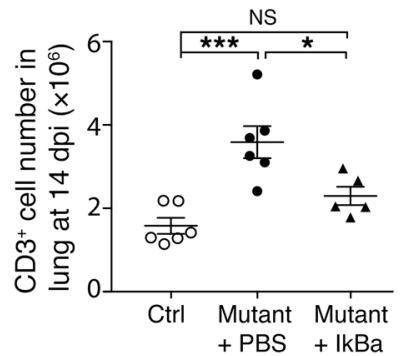

O SPC-CreERT2

Rosa26-mTmG (ctrl)

- SPC-CreERT2: Yap/Taz mutant, Rosa26-mTmG (mutant) + PBS

$\triangle$ SPC-CreERT2: Yap/Taz mutant, Rosa26-mTmG (mutant) + IkBa

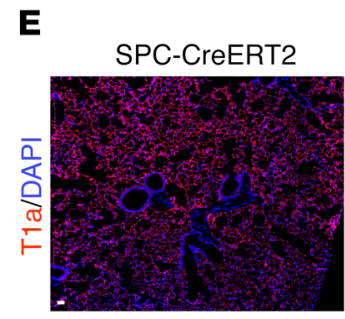

SPC-CreERT2

SPC-CreERT2
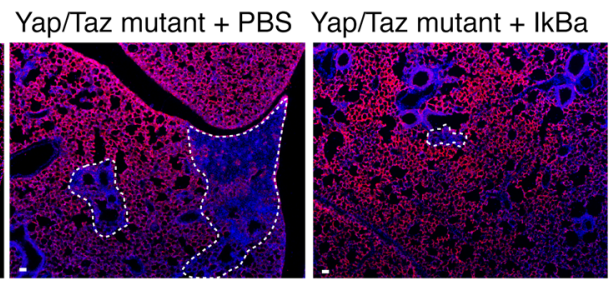

F

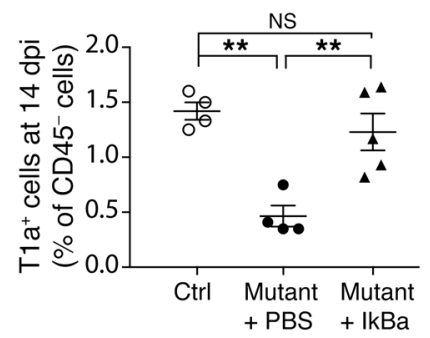

$\circ$

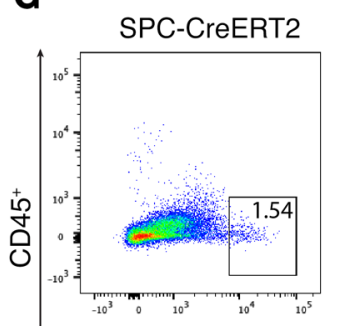

SPC-CreERT2

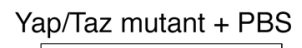

SPC-CreERT2

Yap/Taz mutant + IkBa

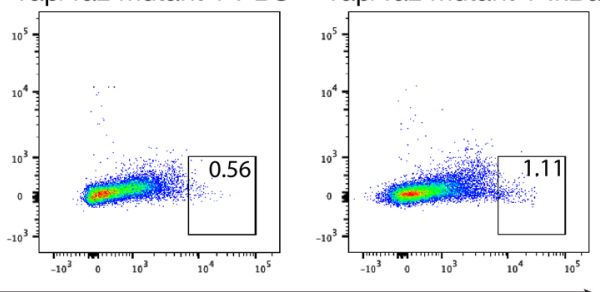

$\mathrm{T} 1 \alpha^{+}$

\section{I} SPC-CreERT2

SPC-CreERT2

SPC-CreERT2

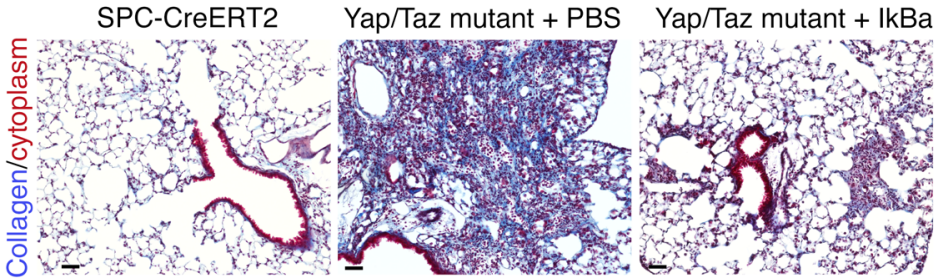

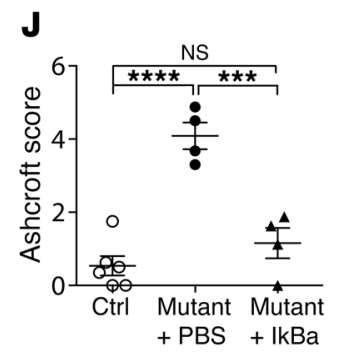

H

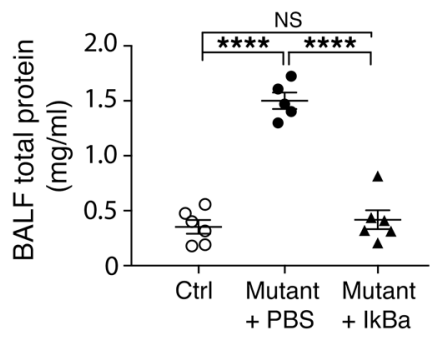

Figure 7. Inflammatory resolution and alveolar epithelial recovery in Yap/Taz mutant lungs with AAV6-IкBa treatment. (A) Schematic of experimental design for B-K. (B) BALF was analyzed for IL-1b by ELISA assay ( $n=4-5$ per group). (C) Cytokine assay showed protein levels of IL-1b and CXCL9 in mouse lung lysates ( $n=1$ per group). (D) Flow cytometry of dissociated lung cells was performed by gating on CD $3^{+} C D 45^{+}$cells, and quantification of total number of $\mathrm{CD3}^{+} \mathrm{CD}_{4} 5^{+}$cells in the lung at $14 \mathrm{dpi}$ were graphed ( $n=5-6$ per group). (E) Immunostaining on lung sections at 14 dpi with nuclei labeled by DAPI (blue) and antibody to T1a (red). White dashed lines indicate T1a- region in the lung. (F) Flow cytometry of dissociated lung cells was performed by gating on T1a+CD45- cells. (G) Quantification of the percentage of T1a+CD45- cells of total CD45- cells in the lung at 14 dpi ( $n=4-5$ per group). (H) Quantification of total protein in BALF at $14 \mathrm{dpi}(n=5-6$ per group). (I) Lung sections at $14 \mathrm{dpi}$ were stained with Alcian blue and nuclear fast red. (J) Quantification of lung fibrotic regions at $14 \mathrm{dpi}$ using Ashcroft scoring method ( $n=4-6$ per group). (K) Quantification of collagen level on lung tissue lysates at $14 \mathrm{dpi}$ using hydroxyproline assay ( $n=4$ per group). ${ }^{*} P<0.05 ;{ }^{* *} P<0.01$; ${ }^{* * *} P<0.001$; ${ }^{* * *} P<0.0001$, 1-way ANOVA. Scale bars: $50 \mu \mathrm{m}$. 
chemokines under appropriate stimulation, showing their role in modulating immunologic activity in the alveolar space. Given the important contributions of AECIIs in lung repair and regeneration, it is critical to determine the molecular mechanisms underlying AECII activities in intact tissues. We showed in this study that Yap and Taz expression and nuclear activity increased in AECIIs upon pneumococcal infection. Yap and Taz regulated AECII activities, including their proliferation, differentiation into AECIs, and inflammatory responses during both homeostasis and lung regeneration following bacterial pneumonia-induced lung injury.

Yap and its closely related paralogue Taz are the transcriptional coactivators that are the main downstream mediators of the Hippo pathway. When Hippo signaling is inhibited, Yap and Taz (Yap/Taz) can accumulate in the nucleus where they interact with transcription factors such as the TEA domain family members (TEAD) and activate gene expression associated with cell survival, proliferation, and differentiation (16). Recent studies have shown the important role of the Hippo/Yap pathway in controlling lung epithelial progenitor cell differentiation during embryogenesis and basal stem cell maintenance in the upper airway of adult lung (26-28). Furthermore, studies of pneumonectomy-induced alveolar regeneration show that Yap is essential for AECII proliferation and differentiation in response to mechanical tension in the lung (29). We measured both AECII proliferation and AECII-to-AECI differentiation in the bacterial pneumonia mouse model. Our data are consistent with previous studies demonstrating the importance of Yap/Taz for the proliferation and differentiation of lung epithelial progenitor cells.

This study demonstrates what we believe is a previously unknown function for Yap/Taz in negative regulation of AECIIderived inflammation. We found that Gram-positive pathogen Streptococcus stimulation of AECIIs to effect inflammatory cytokine/chemokine production required the activation of Yap/TazI $\mathrm{Ba}$, a process that had been previously reported in Drosophila fat cells, both in vitro and in vivo (20). A potential mechanism for Gram-positive pathogen-dependent activation of Yap/Taz-IкB may be the TLR-mediated antimicrobial response (20). The inhibitory $\mathrm{I} \kappa \mathrm{B}$ proteins have been discovered as fundamental regulators of the inducible transcription factor NF- $\kappa \mathrm{B}(30)$. I $\kappa$ Ba preferentially binds to heterodimers containing p50, p65, and c-Rel (31-34). Several comparative studies have demonstrated that IкBa is the

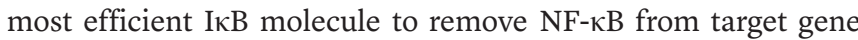
promoters and to terminate $\mathrm{NF}-\kappa \mathrm{B}$-mediated gene induction (35-38). Generally, various stimuli such as TLR ligands triggers

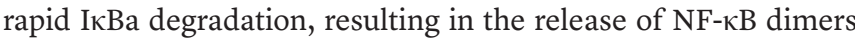
and subsequent transcriptional activation $(30,39,40)$. We found IкBa was transcriptionally regulated by Yap/Taz-Tead in AECIIs. Thus, Yap/Taz-dependent functions are associated with the termination of NF- $\mathrm{KB}$-dependent transcription of inflammatory genes through the induction of IкBa expression. Loss of Yap/Taz removed a "brake" on AECII activation and increased expression of inflammatory genes, leading to elevated local inflammation and enhanced accumulation and persistence of inflammatory cells in the lung (Supplemental Figure 9).

Higher inflammatory responses in pneumococci-infected lungs have been previously reported $(12,13,41)$, but prior work did not examine immune responses in vivo that could affect lung regeneration. Another study found increased AECII proliferation in mice following pneumonectomy, which was correlated with increased monocyte and macrophage recruitment and an increased inflammatory state in the lung (11). They further showed that loss of recruited monocytes/macrophages impaired lung regeneration (11). However, the impact of prolonged inflammation on lung regeneration has not been clear. There is a growing understanding that overly exuberant or persistent inflammatory responses can be found in fibrotic lungs (42-44). Our observations of increased IL-1b, Cxcl3, and Ccl21a expression in the fibrotic lungs of Yap/Taz mutants are consistent with a more inflammatory milieu during bacterial pneumonia. We found that $\mathrm{SPC}^{+} \mathrm{AECIIs}$ required Yap/Taz to active I $\kappa \mathrm{Ba}$, which inhibited NF- $\kappa \mathrm{B}-$ mediated inflammation. Loss of Yap/Taz in $\mathrm{SPC}^{+}$AECIIs led to persistent lung inflammation and fibrotic lesions in lung alveoli during bacterial pneumonia, indicative of impaired alveolar regeneration. We found no Yap/Taz-dependent defects in clearing pneumococcal load in vivo, leading us to the conclusion that it is a role for Yap/ Taz in the resolution of lung inflammation through regulation of $\mathrm{NF}-\kappa \mathrm{B}$ nuclear activity that underlies alveolar regeneration.

During development, epithelial NF- $\kappa \mathrm{B}$ signaling is known to regulate lung inflammation and alveolar development in both humans and mice (45-47). In adulthood, correlations between persistent lung inflammation and decreased lung regeneration have been observed in both various animal injury models and human patients $(8,10)$, but it has not been clear whether higher inflammatory burdens in the lung precede loss of lung regeneration or are an effect of respiratory disease. In this work, we found that loss of Yap/Taz expression in $\mathrm{SPC}^{+}$AECIIs, by promoting constitutive nuclear activity of $\mathrm{NF}-\kappa \mathrm{B}$, led to persistent inflammatory responses in lung alveoli. We also found that restoration of the Yap/Taz target I $\kappa \mathrm{Ba}$, the inhibitor of NF- $\kappa \mathrm{B}$, promoted resolution of lung inflammation and alveolar epithelial recovery from bacterial pneumonia in Yap/Taz mutant mice. Recent studies have reported alveolar regenerative capacity following injury, which is mediated by several stem/progenitor cell populations, including basal cells, club cells, AECIIs and lineage-negative progenitors or distal airway stem cells, and AECIs (10, 48-54). Our work showed that IкBa treatment did not improve AECII-to-AECI differentiation in Yap/Taz mutant lungs, suggesting that regenerated AECIs were contributed by non-SPC+AECIIs. These results emphasize the importance of cellular niche in considering how inflammatory signaling influences adult lung regeneration.

The resolution of inflammation in the lung is a highly controlled and coordinated process that involves the suppression of inflammatory gene expression and inflammatory cell clearance. We highlight the role of the endogenous antiinflammatory mechanism of Yap/Taz that limits the excessive and prolonged production of inflammatory mediators in AECIIs. In general, the onset of inflammation is associated with secretion of cytokines/chemokines to attract circulating immune cells to the site of injury to counteract the infected agent in the affected lung tissue. The activated AECIIs directed the inflammatory processes toward resolution after the tasks of immune cells are successfully accomplished. However, in pathologic chronic inflammation, the inflammatory processes are not resolved, and active inflammation continues in a dysregulated fashion. The persistent lung inflammatory processes 
in Yap/Taz mutants suggest that inappropriate Yap/Taz and IкBa/ NF- $\kappa$ B signaling pathways may also be the major contributors that cause failed regeneration in pathologic chronic lung disease.

\section{Methods}

Mice. All mice were bred and raised in the pathogen-free mouse facility at Temple University. C57BL/6 mice (Jackson Laboratories) and SPC-CreERT2 mice (52) and Yap ${ }^{\mathrm{fl}}$, Taz ${ }^{\mathrm{fl}}$ (55), and Rosa26-mTmG mice (Jackson Laboratories) were used. Generation and genotyping of the SPC-CreERT2, Rosa26-mTmG, Yap ${ }^{\mathrm{fl}}$, and Taz ${ }^{\mathrm{fl}}$ lines have been previously described $(52,55)$. SPC-CreERT2 mice, Yap ${ }^{\mathrm{f} / \mathrm{fl}}$ mice, Taz ${ }^{\mathrm{f} / \mathrm{fl}}$ mice and Rosa26-mTmG mice were kept on a mixed C57BL/6:129SVJ background. All experiments used 6- to 10-week-old male mice.

Bacterial infection. The pneumococcal strain used was the clinical isolated strain SpT4 (56). SpT4 was stored at $-80^{\circ} \mathrm{C}$ and grown in tryptic soy agar plus catalase $(57 \mu \mathrm{gP} / \mathrm{ml})$ under microaerophilic conditions for $14-16$ hours at $37^{\circ} \mathrm{C}$ with $5 \% \mathrm{CO}_{2}$, then subcultured and grown to an optical density of 0.5 . The broth was centrifuged, and the bacteria were washed in sterile PBS and resuspended in $4 \mathrm{ml}$ of sterile PBS immediately prior to infection. Mice were anesthetized using ketamine/xylazine mixture and infected i.n. with a dose of approximately $5 \times 10^{6} \mathrm{CFU}$ in $30 \mu \mathrm{l}$ of sterile PBS.

Bacterial loads. Pneumococcal loads were determined by homogenization of lung tissue in sterile PBS and blood serum at 1, 4, 7, and 14 days after infection of the animals. Tissue homogenate $(100 \mu \mathrm{l})$ or blood serum $(50 \mu \mathrm{l})$ and further dilutions were plated on tryptic soy agar plates plus catalase $(57 \mu \mathrm{gP} / \mathrm{ml})$ for culture overnight at $37^{\circ} \mathrm{C}$ with $5 \% \mathrm{CO}_{2}$, and the number of CFU was counted.

Quantification of lung damage. Lung damage was assessed in BALF. Lungs were lavaged with $1 \mathrm{ml}$ of PBS. BALF was recovered and centrifuged at $1,650 \mathrm{~g}$ for 3 minutes at $4^{\circ} \mathrm{C}$ and supernatant collected. Protein levels were quantified by Pierce BCA protein assay (Thermo Scientific) per the manufacturer's instructions. All plates were read on a Safire II plate reader (Tecan).

Tissue harvest and immunostaining for histology. Mice were euthanized by overdose of tribromoethanol (Avertin, $300 \mathrm{mg} / \mathrm{kg}$ ) followed by cervical dislocation. After exposing the heart and lungs, the descending aorta was cut and a 25-gauge needle was inserted into the right ventricle to flush at least $10 \mathrm{ml}$ of PBS until the color of lungs changed to pink/white. A tracheostomy was performed on the exposed trachea and a 23-gauge stub needle tip was placed into the trachea. The lungs were inflated with $4 \%$ paraformaldehyde (PFA) at a pressure of $2 \mathrm{O} \mathrm{cm} \mathrm{H}_{2} \mathrm{O}$. The trachea was then tied off, and intact lungs were immersed in $4 \%$ PFA for 4 hours at $4^{\circ} \mathrm{C}$. Lung lobes were separated and washed with cold PBS overnight. After ethanol dehydration, lungs were embedded in paraffin and sectioned at $6 \mu \mathrm{m}$. To perform immunohistochemical staining, slides were deparaffinized and rehydrated. Tissue sections were then incubated in citrate buffer ( $\mathrm{pH}$ 6.0) for 20 minutes at $95-100^{\circ} \mathrm{C}$, followed by permeabilization and blocking with $0.3 \%$ Triton X-100 and 5\% goat serum or horse serum (for goat primary antibodies) in PBS for 1 hour at room temperature (r.t.). Primary antibodies were diluted in PBS and incubated overnight at $4^{\circ} \mathrm{C}$. Secondary antibodies were diluted in PBS and incubated for 1 hour at r.t. Immunostainings for Yap and Taz protein were performed using a TSA Fluorescein Tyramide Amplification System. EdU staining, TUNEL staining, and DAPI nuclear staining were performed according to the manufacturers' recommendations. Tissue sections were washed $3 \times$ with PBS between antibody incubations for 15 minutes each. Slides were mounted with Aqua-Poly/Mount (Polysciences Inc.), and images were captured on a Zeiss LSM 710 confocal microscope and a Nikon eclipse fluorescence microscope. Images were processed with ImageJ/ FIJI (NIH). Quantitation of cell numbers was completed using at least 10 randomly selected images per animal. Reagents used in this study are provided in Supplemental Table 3.

Lung dissociation for flow cytometry. Alveolar epithelial cells were isolated using previously described protocols (57). Briefly, after lungs were cleared of blood by perfusion with PBS, as described for the tissue harvest procedure above, $3 \mathrm{ml}$ of dispase $\left(25 \mathrm{U} / \mathrm{ml}, 37^{\circ} \mathrm{C}\right)$ was instilled into the lungs through the trachea followed with instillation of 0.5 $\mathrm{ml}$ of $1 \%$ low melting agarose into the lungs. Agarose was hardened by adding ice on top of the lungs for 2 minutes. Lung lobes were then separated and incubated in $1 \mathrm{ml}$ of dispase for 6 minutes at $37^{\circ} \mathrm{C}$. Each lung lobe was minced in DMEM containing penicillin $\mathrm{G}$, streptomycin, L-glutamine, HEPES, and DNase I $(120 \mathrm{U} / \mathrm{ml})$, followed by a rotating incubation for 10 minutes at r.t. The cells were then filtered sequentially through 100 and $40 \mu \mathrm{m}$ strainers and centrifuged. The cells were incubated with red blood cell lysis buffer for 1 minute on ice and washed with PBS containing 5\% BSA and 0.5 M EDTA. For immune cell flow cytometry, lungs were dissociated with a collagenase/DNase I solution, as previously described $(11,12)$. Lung lobes were cut into small pieces by scalpel blade and then incubated in $5 \mathrm{ml}$ of digestion solution containing collagenase type I ( $3 \mathrm{mg} / \mathrm{ml})$, DNase I ( $80 \mathrm{U} / \mathrm{ml}), 10 \% \mathrm{FBS}$, and $2-\mathrm{ME}(0,055 \mathrm{mM})$ for 45 minutes at $37^{\circ} \mathrm{C}$. Flow cytometry was performed on a BD LSR II and sorting was performed on a BD INFLUX.

Immunostaining for flow cytometry. Dissociated cells were blocked with $\mathrm{CD} 16 / \mathrm{CD} 32 \mathrm{Fc}$ receptor block at 1:100 for 20 minutes. After washing with PBS, cells were incubated with fluorophore-conjugated antibodies for 1 hour. For live cell staining, LIVE/DEAD Fixable Aqua Dead Cell Stain Kit was added along with fluorophore-conjugated antibody mix at a 1:1000 dilution. Cells were washed twice with PBS, placed in FACS buffer containing 1\% BSA and 0.05\% sodium azide in PBS, and then assessed using a LSR II (BD). Analysis was performed using FlowJo (10.4.2).

Gene expression and microarray analysis. qPCR analysis was performed using TRIzol-isolated RNA, which was used to generate cDNA using random hexamer primers and SuperScript III RT. Primer sequences are listed in Supplemental Table 4. For microarray analysis, total RNA was extracted from 6- to 8-week-old SPC-CreERT2, Rosa26-mTmG GFP ${ }^{+}$cells at 0 and $8 \mathrm{dpi}$, and SPC-CreERT2, Yap ${ }^{\mathrm{f} / \mathrm{fl}}$, $\mathrm{Taz}^{\mathrm{f} / \mathrm{fl}}$, Rosa26-mTmG GFP ${ }^{+}$cells at $8 \mathrm{dpi}$, converted to cDNA and used on Affymetrix Mouse Gene 1.0 ST arrays. Data were analyzed using Affymetrix Microarray Suite 5.0, Significance Analysis of Microarray (SAM), and the Empirical Bayes Analyses of Microarrays (EBAM). Genes with 1.5-fold or greater changes over that of the experimental mean at $P<0.01$ (ANOVA) were considered significant. All original microarray data were deposited in the NCBI's Gene Expression Omnibus database (GEO GSE86027). Hierarchical clustering was performed using Gene Cluster 3.0. Gene Ontology (GO) associations and related $P$ values were determined using the ToppGene Suite (58). (https://toppgene.cchmc.org)

Western blot. For extraction of proteins from AECIIs, lungs were dissociated with a dispase solution, as described in the procedure of lung dissociation for flow cytometry above. AECIIs were further purified using magnetic beads and column isolation, as previously 
described (58). Briefly, cells were incubated with CD45 Microbeads for 15 minutes at $4^{\circ} \mathrm{C}$ and washed with cold column buffer (PBS containing $5 \%$ BSA and 0.5M EDTA). Cells were resuspended in cold column buffer and applied to MACS LS columns on a MACS separator (Miltenyi Biotec). The flow-through cells were blocked with FcR blocking reagent for 10 minutes at $4^{\circ} \mathrm{C}$, followed by an incubation with binotinylated EpCAM antibody for 30 minutes at $4^{\circ} \mathrm{C}$. Cells were washed and incubated with Streptavidin Microbeads for 20 minutes at $4^{\circ} \mathrm{C}$. Cells were resuspended in cold column buffer and applied to MACS LS columns. The columns were then removed from the magnetic separator. The magnetically labeled AECIIs were collected by forcing $5 \mathrm{ml}$ of cold column buffer into the column. After centrifugation, AECIIs were lysed using RIPA buffer containing protease and phosphatase inhibitors. For extraction of proteins from tissues, lungs were homogenized in RIPA buffer using a tissue grinder. Protein concentrations were determined using the BCA Protein Assay Reagent Kit (Bio-Rad). Protein extracts were analyzed on polyacrylamide gels (10\% NuPAGE Bis-Tris Gel; Invitrogen) and transferred to nitrocellulose membrane (Bio-Rad). The blots were blocked in 5\% BSA in TBS for 1 hour at r.t., followed by incubation with primary antibody diluted in block overnight at $4^{\circ} \mathrm{C}$. The blots were washed $3 \times$ with TBS containing $0.1 \%$ Tween 20 (TBST) for 15 minutes and incubated with near-infrared fluorophore-conjugated secondary antibodies diluted in TBST for 1 hour at r.t. Signals were detected using the Odyssey imaging system (LI-COR Biosciences).

Cell lines, transfections, and treatments. Mouse MLE-15 cells (abm Inc., catalog T0495) were grown in DMEM/F12 containing 2\% FBS, $10 \mathrm{mM}$ HEPES, and $10 \mathrm{nM}$ hydrocortisone. HEK293T cells were purchased from ATCC and grown in DMEM containing 10\% FBS. DNA transfections were done with X-tremeGENE HP DNA Transfection Reagent (Roche) in antibiotic-free medium according to the manufacturer's instructions. Yap shRNA and scrambled shRNA were used as previously described (25). Lentiviral particles were prepared by transiently transfecting HEK293T cells with lentiviral vectors together with packaging vectors (pMD2-VSVG and psPAX2).

Plasmids. Murine Yap was described previously (59) and subcloned into the pCMV-Tag3B vector. Murine Taz was expressed in the pCMVSport6 vector (Addgene plasmid 27318). YAP-5SA and TAZ-S89A plasmids were purchased from Addgene (plasmid 27371 and 32840). Murine Tead 2 cDNA was purchased from OriGene (catalog MR223302) and subcloned into the pcDNA3.1 $\left(^{+}\right)$vector. NF- $\kappa$ B response luciferase reporter was purchased from Promega (catalog E8491). A 1900 bp region of the murine IкBa, gene enhancer ( -8600 to approximately $-6700 \mathrm{~kb}$ relative to the ATG site) was cloned into a pGL3 luciferase reporter vector (Promega, GenBank, catalog U47298). Point mutations within the IкBa, enhancer-luciferase reporter plasmid were generated using the QuikChange Site-Directed Mutagenesis Kit (Stratagene). Sequences of cloning primers are provided in Supplemental Table 4.

ChIP quantitative PCR. ChIP assays were performed using the EZ-Magna ChIP A/G Chromatin Immunoprecipitation Kit (Millipore). An equal amount of chromatin $\left(1 \times 10^{6}\right.$ cells $)$ was used for each precipitation. Cells were crosslinked with $1 \%$ formaldehyde for 10 minutes at r.t., and the reaction was quenched with the addition of $0.14 \mathrm{M}$ glycine for 5 minutes at r.t. Cell pellets were resuspended in SDS lysis buffer (10 mM Tris- $\mathrm{HCl}, \mathrm{pH}$ 8.0, $10 \mathrm{mM} \mathrm{NaCl}, 3 \mathrm{mM} \mathrm{MgCl}, 1 \% \mathrm{NP}-40,1 \%$ SDS, $0.5 \%$ deoxycholic acid, and protease inhibitors). Chromatin was sheared using a sonicator (Vibracell) on ice and then processed for antibody incubation overnight at $4^{\circ} \mathrm{C}$. The following antibodies $(0.5$ $\mu \mathrm{g})$ were used: Tead, Yap, and control IgG. Protein A/G agarose beads were added to each reaction and incubated for 1 hour at $4^{\circ} \mathrm{C}$. The agarose beads were washed, and chromatin was reverse crosslinked and purified according to the manufacturer's instructions. $4 \%$ Of the precipitated chromatin was used for each PCR reaction. Sequences of the promoter-specific primers are provided in Supplemental Table 4.

Luciferase reporter assay. MLE-15 cells were seeded on 12-well

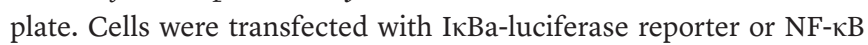
response luciferase reporter together with Renilla luciferase reporter plasmid and either the Tead2, Yap, or Taz plasmid. After 24 hours, cells were lysed and processed for luciferase assay using the Dual Luciferase Assay System (Promega).

Preparation of nuclear extracts and cell lysates. AECIIs $\left(5 \times 10^{6}\right)$ were centrifuged at $200 \mathrm{~g}$ for 5 minutes, and the pellet was suspended in 250 $\mu \mathrm{l}$ of hypotonic buffer (10 mM HEPES, pH 7.9, 0.1 mM EDTA, $10 \mathrm{mM}$ $\mathrm{KCl}$, protease and phosphatase inhibitor cocktail solution, $1 \times$ ). Cells were incubated at $4^{\circ} \mathrm{C}$ for 15 minutes, which was followed by adding $12.5 \mu \mathrm{l}$ of $10 \% \mathrm{NP} 40$ and vortexing for 10 seconds. After centrifuging at $6,000 \mathrm{~g}$ at $4^{\circ} \mathrm{C}$ for 10 minutes, cell pellets were suspended in $50 \mu \mathrm{l}$ of nuclear extraction buffer (10 mM HEPES-KOH, pH 7.9, 10\% glycerol, $420 \mathrm{mM} \mathrm{NaCl}, 1.5 \mathrm{mM} \mathrm{MgCl}_{2}, 0.1 \mathrm{mM}$ EDTA, protease and phosphatase inhibitor cocktail solution, $1 \times$ ). Nuclei were lysed by incubating at $4^{\circ} \mathrm{C}$ for 30 minutes, with vortexing for 15 seconds at 10 -minute intervals. Lysates were centrifuged at $14,000 \mathrm{~g}$ for 10 minutes at $4^{\circ} \mathrm{C}$. Supernatants were collected, and the protein concentrations were measured using the BCA assay. Nuclear protein aliquots were stored at $-80^{\circ} \mathrm{C}$.

Protein cytokine array. Whole-lung left lobes were isolated and flash frozen in liquid nitrogen. Tissue was homogenized in $2 \mathrm{ml}$ PBS per lobe containing cOmplete Mini protease inhibitor cocktail (MilliporeSigma). Following homogenization, Triton X-100 was added to a final concentration of $1 \%$, then frozen at $-20^{\circ} \mathrm{C}$ overnight. Samples were thawed on ice and centrifuged at 10,000 $g$ for 5 minutes, and supernatants were collected. A standardized BCA protein assay was performed to measure lysate protein concentration (Thermo Fisher Scientific, catalog 23225). Purified protein lysates $(300 \mathrm{mg}$ ) were applied to the Proteome Profiler Mouse Cytokine Array Kit, Panel A (R\&D Systems, catalog ARY006). The chemiluminescence reaction was measured with a Konica Minolta SRX-101A Medical Film Processor (code no. 1051/1052). Densitometry quantitation was performed in FIJI/ImageJ (NIH).

Hydroxyproline assay. Lungs were homogenized in $1 \mathrm{ml}$ of PBS and then hydrolyzed in $6 \mathrm{~N} \mathrm{HCl}$ at $120^{\circ} \mathrm{C}$ for 16 hours. After centrifugation at $10,000 \mathrm{~g}$ for 5 minutes, the supernatant was transferred to a new tube and then processed for dryness by heating the tube at $65^{\circ} \mathrm{C}$ on a hot plate overnight. Oxidation reagent mix $(100 \mu \mathrm{l}, 14 \%$ chloramine $\mathrm{T}$, $0.24 \mathrm{M}$ citric acid monohydrate, $0.2 \mathrm{M}$ acetic acid glacial, $0.53 \mathrm{M}$ sodium acetate trihydrate, $0.85 \mathrm{M}$ sodium hydroxide) was added to the crystalline residue in the tube. After incubation at r.t. for 20 minutes, $100 \mu \mathrm{l}$ of Erlich's solution was added to the mixture and then processed for 15 -minute incubation at $65^{\circ} \mathrm{C}$. Absorbance was measured at $550 \mathrm{~nm}$.

Ashcroft scoring method of estimating severity of pulmonary fibrosis. Ashcroft scores were quantified as previously described (60). Eight images per sample at various parts of each lung were taken and their fibrosis score averaged to get a fibrosis score for the sample as a whole. Each image was graded individually based on the Ashcroft criteria. For images that had areas of fibrosis and areas of normal lung tissue, a score was given to each section and then averaged to yield the fibrosis score of the image. 
Intratracheal instillation in mice. The procedure was performed as previously described (61). Mice were anesthetized by i.p. injection of ketamine/xylazine mixture, and AAV6-IкBa or AAV6-GFP or AAV6null diluted in $50 \mathrm{ul}$ of sterile PBS was instilled intratracheally via a 23 -gauge cannula. The dose was $2.5 \times 10^{10} \mathrm{vp} /$ animal.

Statistics. Data are reported as mean \pm SEM. Multiple groups were compared by 1-way ANOVA followed Tukey's or Dunnett's post hoc test. Multiple groups with multiple time points were compared by 2-way ANOVA, followed by Šidák's multiple comparisons and post hoc tests. Student's $t$ test was used when comparing 2 experimental groups. A $P$ value of less than 0.05 was considered significant. $P>0.05$ was considered not significant. All analyses were performed with GraphPad Prism 7 .

Study approval. This study was conducted according to the guidelines outlined by the Public Health Service Policy on the Human Care and Use of Laboratory Animals. All protocols for breeding and experiments with animals were approved by the IACUC at Temple University, protocol 4732.

\section{Author contributions}

RL performed the majority of experiments and participated in writing the manuscript. DL and PZ assisted RL in mouse lung AECII isolation and immunostaining. LT performed lung fibrosis analy- sis, plasmid cloning, and luciferase reporter assay. YW performed SpT4 infection on mice. TC performed histology. HAC and EEM advised on the SPC-CreERT2 animal model and edited the manuscript. HS advised on the SpT4 infection model. WJK advised on AAV6 treatment of mice. BK advised on AECII isolation and culture. MRW advised on intratracheal instillation on mice. YT supervised all experiments and wrote the manuscript.

\section{Acknowledgments}

We thank Eric N. Olson for sharing Yap $^{\mathrm{fl}}$ and $\mathrm{Taz}^{\mathrm{fl}}$ mice. We thank Joseph Rabinowitz for providing AAV6-Yap-5SA, AAV6-TazS89A, AAV6-IкBa, and AAV6-GFP. Yap-5SA and Taz-S89A plasmids were gifts from Kunliang Guan (Addgene plasmid 27371 and 32840). Murine Yap cDNA plasmid was a gift from Seo Hee Cho and Seonhee Kim. pSport6-flag-Taz plasmid was a gift from Jonathan Epstein (Addgene plasmid 27318). YT is supported by R00HL111348 and RO1-HL132115. BK is supported by RO1-HL118171.

Address correspondence to: Ying Tian, Department of Pharmacology, Center for Translational Medicine, Temple University Lewis Katz School of Medicine, Philadelphia, Pennsylvania 19140, USA. Phone: 215.707.7192; Email: ying.tian@temple.edu.
1. O'Brien KL, et al. Burden of disease caused by Streptococcus pneumoniae in children younger than 5 years: global estimates. Lancet. 2009;374(9693):893-902

2. Bhowmick R, et al. Systemic disease during Streptococcus pneumoniae acute lung infection requires 12-lipoxygenase-dependent inflammation. JImmunol. 2013;191(10):5115-5123.

3. Hogan BL, et al. Repair and regeneration of the respiratory system: complexity, plasticity, and mechanisms of lung stem cell function. Cell Stem Cell. 2014;15(2):123-138.

4. Barkauskas CE, et al. Type 2 alveolar cells are stem cells in adult lung. JClin Invest. 2013;123(7):3025-3036.

5. Fehrenbach H. Alveolar epithelial type II cell: defender of the alveolus revisited. Respir Res. 2001;2(1):33-46.

6. Manicone AM. Role of the pulmonary epithelium and inflammatory signals in acute lung injury. Expert Rev Clin Immunol. 2009;5(1):63-75.

7. Todd NW, Luzina IG, Atamas SP. Molecular and cellular mechanisms of pulmonary fibrosis. Fibrogenesis Tissue Repair. 2012;5(1):11.

8. Kawanami O, Ferrans VJ, Crystal RG. Structure of alveolar epithelial cells in patients with fibrotic lung disorders. Lab Invest. 1982;46(1):39-53.

9. Kasper M, Haroske G. Alterations in the alveolar epithelium after injury leading to pulmonary fibrosis. Histol Histopathol. 1996;11(2):463-483.

10. Vaughan AE, et al. Lineage-negative progenitors mobilize to regenerate lung epithelium after major injury. Nature. 2015;517(7536):621-625.

11. Lechner AJ, et al. Recruited monocytes and type 2 immunity promote lung regeneration following pneumonectomy. Cell Stem Cell. 2017;21(1):120-134.e7.

12. Wang Y, et al. Cross-protective mucosal immunity mediated by memory Th17 cells against Streptococcus pneumoniae lung infection. Mucosal
Immunol. 2017;10(1):250-259.

13. Malley R, et al. Antibody-independent, interleukin-17A-mediated, cross-serotype immunity to pneumococci in mice immunized intranasally with the cell wall polysaccharide. Infect Immun. 2006;74(4):2187-2195.

14. Karin M, Clevers H. Reparative inflammation takes charge of tissue regeneration. Nature. 2016;529(7586):307-315.

15. Eming SA, Wynn TA, Martin P. Inflammation and metabolism in tissue repair and regeneration. Science. 2017;356(6342):1026-1030.

16. Yu FX, Guan KL. The Hippo pathway: regulators and regulations. Genes Dev. 2013;27(4):355-371.

17. Varelas X. The Hippo pathway effectors TAZ and YAP in development, homeostasis and disease. Development. 2014;141(8):1614-1626.

18. Sims JE, Smith DE. The IL-1 family: regulators of immunity. Nat Rev Immunol. 2010;10 (2):89-102.

19. Ochiai E, et al. CXCL9 is important for recruiting immune $\mathrm{T}$ cells into the brain and inducing an accumulation of the T cells to the areas of tachyzoite proliferation to prevent reactivation of chronic cerebral infection with Toxoplasma gondii. Am J Pathol. 2015;185(2):314-324.

20. Liu B, Zheng Y, Yin F, Yu J, Silverman N, Pan D. Toll receptor-mediated hippo signaling controls innate immunity in Drosophila. Cell. 2016;164(3):406-419.

21. Simeonidis S, Liang S, Chen G, Thanos D. Cloning and functional characterization of mouse IkappaBepsilon. Proc Natl Acad Sci U S A. 1997;94(26):14372-14377.

22. Malek S, Chen Y, Huxford T, Ghosh G. IkappaBbeta, but not IkappaBalpha, functions as a classical cytoplasmic inhibitor of NF-kappaB dimers by masking both NF-kappaB nuclear localization sequences in resting cells. J Biol Chem. 2001;276(48):45225-45235.

23. Tran K, Merika M, Thanos D. Distinct functional properties of IkappaB alpha and IkappaB beta Mol Cell Biol. 1997;17(9):5386-5399.

24. Skerrett SJ, Liggitt HD, Hajjar AM, Ernst RK, Miller SI, Wilson CB. Respiratory epithelial cells regulate lung inflammation in response to inhaled endotoxin. Am J Physiol Lung Cell Mol Physiol. 2004;287(1):L143-L152.

25. Tian Y, et al. A microRNA-Hippo pathway that promotes cardiomyocyte proliferation and cardiac regeneration in mice. Sci Transl Med. 2015;7(279):279ra38.

26. Lange AW, Sridharan A, Xu Y, Stripp BR, Perl AK, Whitsett JA. Hippo/Yap signaling controls epithelial progenitor cell proliferation and differentiation in the embryonic and adult lung. J Mol Cell Biol. 2015;7(1):35-47.

27. Mahoney JE, Mori M, Szymaniak AD, Varelas X, Cardoso WV. The hippo pathway effector Yap controls patterning and differentiation of airway epithelial progenitors. Dev Cell. 2014;30(2):137-150.

28. Zhao R, et al. Yap tunes airway epithelial size and architecture by regulating the identity, maintenance, and self-renewal of stem cells. Dev Cell. 2014;30(2):151-165.

29. Liu Z, et al. MAPK-mediated YAP activation controls mechanical-tension-induced pulmonary alveolar regeneration. Cell Rep. 2016;16(7):1810-1819.

30. Hinz M, Arslan SÇ, Scheidereit C. It takes two to tango: I $\kappa s$, the multifunctional partners of NF-кB. Immunol Rev. 2012;246(1):59-76.

31. Brown K, Park S, Kanno T, Franzoso G, Siebenlist U. Mutual regulation of the transcriptional activator NF-kappa B and its inhibitor, I kappa B-alpha. Proc Natl Acad Sci U S A. 1993;90(6):2532-2536.

32. Sun SC, Ganchi PA, Ballard DW, Greene WC. NF-kappa B controls expression of inhibitor I kappa B alpha: evidence for an inducible autoregulatory pathway. Science. 1993;259(5103):1912-1915. 33. de Martin R, et al. Cytokine-inducible expres- 
sion in endothelial cells of an I kappa B alphalike gene is regulated by NF kappa B. $Е M B O J$. 1993;12(7):2773-2779.

34. Le Bail O, Schmidt-Ullrich R, Israël A. Promoter analysis of the gene encoding the I kappa Balpha/MAD3 inhibitor of NF-kappa B: positive regulation by members of the rel/NF-kappa B family. EMBO J. 1993;12(13):5043-5049.

35. Zabel U, Baeuerle PA. Purified human I kappa $\mathrm{B}$ can rapidly dissociate the complex of the NF-kappa B transcription factor with its cognate DNA. Cell. 1990;61(2):255-265.

36. Hatada EN, et al. The ankyrin repeat domains of the NF-kappa B precursor p105 and the protooncogene bcl-3 act as specific inhibitors of NFkappa B DNA binding. Proc Natl Acad Sci U S A. 1992;89(6):2489-2493.

37. Beg AA, Ruben SM, Scheinman RI, Haskill S, Rosen CA, Baldwin AS. I kappa B interacts with the nuclear localization sequences of the subunits of NF-kappa B: a mechanism for cytoplasmic retention. Genes Dev. 1992;6(10):1899-1913.

38. Nolan GP, Ghosh S, Liou HC, Tempst P, Baltimore D. DNA binding and I kappa B inhibition of the cloned $\mathrm{p} 65$ subunit of NF-kappa B, a relrelated polypeptide. Cell.1991;64(5):961-969.

39. Hayden MS, Ghosh S. Shared principles in NF-kappaB signaling. Cell. 2008;132(3):344-362.

40. Vallabhapurapu S, Karin M. Regulation and function of NF-kappaB transcription factors in the immune system. Annu Rev Immunol. 2009;27:693-733.

41. Paterson GK, Mitchell TJ. Innate immunity and the pneumococcus. Microbiology (Reading, Engl). 2006;152(Pt 2):285-293.

42. Birjandi SZ, et al. CD4(+)CD25(hi)Foxp3(+) cells exacerbate bleomycin-induced pulmonary fibro- sis. Am J Pathol. 2016;186(8):2008-2020.

43. Herazo-Maya JD, et al. Peripheral blood mononuclear cell gene expression profiles predict poor outcome in idiopathic pulmonary fibrosis. Sci Transl Med. 2013;5(205):205ra136.

44. Reilkoff RA, et al. Semaphorin 7a+ regulatory $\mathrm{T}$ cells are associated with progressive idiopathic pulmonary fibrosis and are implicated in transforming growth factor- $\beta 1$-induced pulmonary fibrosis. Am J Respir Crit Care Med. 2013;187(2):180-188.

45. Benjamin JT, et al. Epithelial-derived inflammation disrupts elastin assembly and alters saccular stage lung development. Am J Pathol. 2016;186(7):1786-1800.

46. Bourbia A, Cruz MA, Rozycki HJ. NF-kappaB in tracheal lavage fluid from intubated premature infants: association with inflammation, oxygen, and outcome. Arch Dis Child Fetal Neonatal Ed. 2006;91(1):F36-F39.

47. Ali S, et al. Functional genetic variation in NFKBIA and susceptibility to childhood asthma, bronchiolitis, and bronchopulmonary dysplasia. JImmunol. 2013;190(8):3949-3958.

48. Kumar PA, et al. Distal airway stem cells yield alveoli in vitro and during lung regeneration following H1N1 influenza infection. Cell. 2011;147(3):525-538.

49. Zuo W, et al. p63(+)Krt5(+) distal airway stem cells are essential for lung regeneration. Nature. 2015;517(7536):616-620.

50. Rawlins EL, et al. The role of Scgb1a1+ Clara cells in the long-term maintenance and repair of lung airway, but not alveolar, epithelium. Cell Stem Cell. 2009;4(6):525-534.

51. Desai TJ, Brownfield DG, Krasnow MA. Alveolar progenitor and stem cells in lung development, renewal and cancer. Nature. 2014;507(7491):190-194.

52. Chapman HA, et al. Integrin $\alpha 6 \beta 4$ identifies an adult distal lung epithelial population with regenerative potential in mice. JClin Invest. 2011;121(7):2855-2862.

53. Jain R, et al. Plasticity of Hopx(+) type I alveolar cells to regenerate type II cells in the lung. Nat Commun. 2015;6:6727.

54. Yee $\mathrm{M}$, et al. Alternative progenitor lineages regenerate the adult lung depleted of alveolar epithelial type 2 cells. Am J Respir Cell Mol Biol. 2017;56(4):453-464.

55. Xin M, et al. Regulation of insulin-like growth factor signaling by Yap governs cardiomyocyte proliferation and embryonic heart size. Sci Signal. 2011;4(196):ra70.

56. Tettelin $\mathrm{H}$, et al. Complete genome sequence of a virulent isolate of Streptococcus pneumoniae. Science. 2001;293(5529):498-506.

57. Messier EM, Mason RJ, Kosmider B. Efficient and rapid isolation and purification of mouse alveolar type II epithelial cells. Exp Lung Res. 2012;38(7):363-373.

58. de Hoon MJ, Imoto S, Nolan J, Miyano S. Open source clustering software. Bioinformatics. 2004;20(9):1453-1454.

59. Park R, et al. Yap is required for ependymal integrity and is suppressed in LPA-induced hydrocephalus. Nat Commun. 2016;7:10329.

60. Ashcroft T, Simpson JM, Timbrell V. Simple method of estimating severity of pulmonary fibrosis on a numerical scale. JClin Pathol. 1988;41(4):467-470.

61. Starcher B, Williams I. A method for intratracheal instillation of endotoxin into the lungs of mice. Lab Anim. 1989;23(3):234-240. 